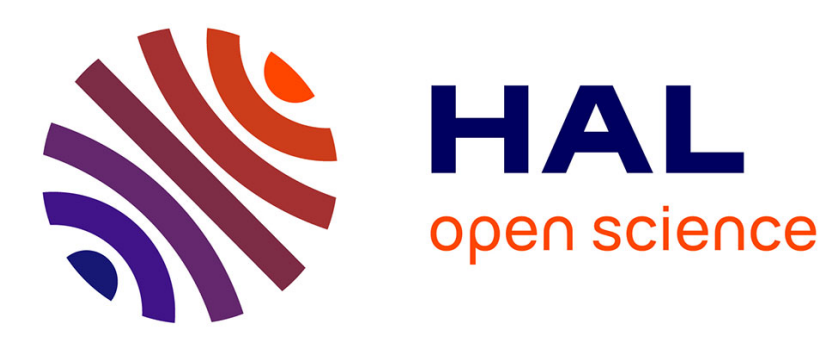

\title{
A simplified fluid-structure model for arterial flow. Application to retinal hemodynamics
}

\author{
Matteo Aletti, Jean-Frédéric Gerbeau, Damiano Lombardi
}

\section{To cite this version:}

Matteo Aletti, Jean-Frédéric Gerbeau, Damiano Lombardi. A simplified fluid-structure model for arterial flow. Application to retinal hemodynamics. Computer Methods in Applied Mechanics and Engineering, 2016, 306, pp.77-94. 10.1016/j.cma.2016.03.044 . hal-01296940

\section{HAL Id: hal-01296940 \\ https://hal.science/hal-01296940}

Submitted on 9 May 2016

HAL is a multi-disciplinary open access archive for the deposit and dissemination of scientific research documents, whether they are published or not. The documents may come from teaching and research institutions in France or abroad, or from public or private research centers.
L'archive ouverte pluridisciplinaire HAL, est destinée au dépôt et à la diffusion de documents scientifiques de niveau recherche, publiés ou non, émanant des établissements d'enseignement et de recherche français ou étrangers, des laboratoires publics ou privés. 


\title{
A simplified fluid-structure model for arterial flow. Application to retinal hemodynamics. ${ }^{1}$
}

\author{
Matteo Aletti, Jean-Frédéric Gerbeau, Damiano Lombardi \\ Inria Paris \& Sorbonne Universités UPMC Univ Paris 6, France
}

\begin{abstract}
We propose a simplified fluid-structure interaction model for applications in hemodynamics. This work focuses on simulating the blood flow in arteries, but it could be useful in other situations where the wall displacement is small. As in other approaches presented in the literature, our simplified model mainly consists of a fluid problem on a fixed domain, with Robin-like boundary conditions and a first order transpiration. Its main novelty is the presence of fibers in the solid. As an interesting numerical side effect, the presence of fibers makes the model less sensitive than others to strong variations or inaccuracies in the curvatures of the wall. An application to retinal hemodynamics is investigated.
\end{abstract}

Keywords:

fluid-structure interaction, blood flow, fibers

\section{Introduction}

Fluid-structure interaction plays an important role in the cardiovascular system. In many situations, complex nonlinear models that include large displacements and deformations have to be considered. This is, for example, the case for valve simulation $[1,2,3,4]$ or in the aorta $[5,6,7]$. It is well-known that these simulations are very demanding, and in spite of the progress achieved in recent years $([8,9,10]$ to name but a few $)$, they remain challenging and the subject of active research.

\footnotetext{
${ }^{1}$ A final version of this manuscript can be found in Computer Methods in Applied Mechanics and Engineering (2016)
} 
In this paper, we consider those situations where it is assumed that the problem under study can be addressed using simplified approaches. The idea is to radically simplify the solid model in order to replace the full fluidstructure problem by a fluid problem with non-standard boundary conditions at the fluid-structure interface. Various approaches have been recently proposed in this direction $[11,12,13,14]$.

In [12], F. Nobile and C. Vergara started from a Koiter linear shell model and neglected the flexural terms. After discretization, the resulting fluidstructure equations are reduced to a fluid problem with Robin boundary conditions. In this approach, the fluid domain was moving. In [14], O. Pironneau further simplified this approach by fixing the fluid domain, introducing a zero order transpiration boundary condition, and by assuming that the curvature of the artery was constant. More precisely, whatever the geometry of the vessel is, the stiffness term is always computed as if the vessel were a cylinder. With these simplifications, the authors were able to perform a comprehensive mathematical analysis of the problem [15]. In [11], A. Figueroa et al. also assumed that the computational domain was fixed and used a zero order transpiration boundary condition. The structural model was derived assuming homogeneity throughout the thickness. Compared to the two previous approaches, this one requires adding new degrees of freedom to the fluid problem. This drawback is, however, counterbalanced by the fact that the resulting model is more stable on real geometries featuring variations of curvature, according to [13] where an extensive comparison was proposed.

The main focus of the present study is the simulation of the autoregulation of blood flow in the retinal arteries. This phenomenon is very important since defective autoregulation may play a role in many retinal diseases, including glaucoma which is the second leading cause of blindness worldwide [16]. Autoregulation consists of an active change of the artery diameter in response to a change in the mean perfusion pressure. This is clearly a fluid-structure interaction problem, but it is typically a case in which a full structural model does not seem necessary, at least to render the basic phenomenon, which is a slight contraction of the vessel wall. The application of the model proposed in this article to the autoregulation of blood flow is presented in more detail in $[17]$.

In our approach, we choose to keep the computational domain fixed, as in [11], and we adopt transpiration boundary conditions. Nevertheless, the phenomenon of autoregulation cannot be addressed with the zero order transpiration formula usually adopted in the literature. Our model will therefore 
be based on first order transpiration. For the structure, we will start from a Koiter shell model without flexural terms as in [12]. But, as noted in [13], this leads to a simplified model that may be unstable in real geometries due to inaccuracies in the curvature obtained from medical images. Even when the curvatures are computed accurately, this model, called inertial-algebraic, may be inaccurate in geometries with a locally flat fluid-structure interface. Note that the model proposed in [14] does not suffer from this problem since it assumes everywhere a cylinder-like geometry in the terms involving the curvature. In our model, we will show that the introduction of fibers allows us to overcome this problem thanks to the presence of a surface Laplace operator.

In summary, the main features of our simplified fluid-structure model are the following: it mainly consists of a fluid problem on a fixed domain, with Robin-like boundary conditions, which makes it insensitive to the added-mass effect; it takes into account in a simplified manner the presence of fibers in the solid; it is less sensitive than others to strong variations or inaccuracies in the curvatures and, as a consequence, it remains robust in the presence of flat regions in the surface.

The structure of the article is as follows: in Section 2 the structure model is proposed; in Section 3 the fluid model and the coupling are presented; Section 4 deals with numerical discretization issues and Section 5 contains numerical illustrations, including an initial simulation in a retinal arteries network.

\section{Structure model}

In this section, a simplified structure model is introduced to describe the dynamics of the wall. Similarly to other studies presented in the literature, the starting point is the Koiter thin shell model (see $[12,14]$ ). The resulting model aims to render the motion of a thin shell with one or several fiber layers. When the kinematics of the fibers is considered, it is relevant to keep second order terms in their deformation because they have an important role in the stability of the model (the gradient terms in equation (5) below). As a consequence, to be consistent with the approximation made for the fibers, second order terms will also be kept in the shell model. This leads to the inclusion of non-linear terms in the shell model. 


\subsection{Notation}

Let $\Gamma$ be the reference position of the structure and a smooth mapping $\phi$ defining its position: $\boldsymbol{\phi}: \omega \subset \mathbb{R}^{2} \rightarrow \Gamma \subset \mathbb{R}^{3}, \boldsymbol{\phi}=\boldsymbol{\phi}\left(\xi_{1}, \xi_{2}\right), \forall\left(\xi_{1}, \xi_{2}\right) \in \omega$. Let $\left(\boldsymbol{a}_{1}, \boldsymbol{a}_{2}\right)$ be the local covariant basis given by $\boldsymbol{a}_{\alpha}=\partial_{\alpha} \boldsymbol{\phi}=\frac{\partial \boldsymbol{\phi}}{\partial \xi_{\alpha}}, \quad \alpha=1,2$. In what follows, Greek letters for the indices take values in $\{1,2\}$ and latin letters in $\{1,2,3\}$. The normal unit vector is defined as $\boldsymbol{a}_{3}=\frac{\boldsymbol{a}_{1} \times \boldsymbol{a}_{2}}{\left|\boldsymbol{a}_{1} \times \boldsymbol{a}_{2}\right|}$. Let $\boldsymbol{A}$ and $\boldsymbol{B}$ be the matrix representations of the first and second fundamental forms associated with the reference configuration $\Gamma$ and let $\boldsymbol{S}=\boldsymbol{A}^{-1} \boldsymbol{B}$ be the representation of the shape operator. The entries of $\boldsymbol{A}$ and $\boldsymbol{B}$ are respectively given by $a_{\alpha \beta}=a_{\beta \alpha}=\boldsymbol{a}_{\alpha} \cdot \boldsymbol{a}_{\beta}$ and $b_{\alpha \beta}=b_{\beta \alpha}=\boldsymbol{a}_{3} \cdot \partial_{\alpha} \boldsymbol{a}_{\beta}$. The entries of $\boldsymbol{A}^{-1}$ are denoted by $a^{\alpha \beta}$, thus $a^{\alpha \sigma} a_{\sigma \beta}=\delta_{\alpha \beta}$. Given a tensor $\boldsymbol{M}=\left(m_{\sigma \beta}\right)$, the entries of $\boldsymbol{A}^{-1} \boldsymbol{M}$ are denoted by $m_{\beta}^{\alpha}=a^{\alpha \sigma} m_{\sigma \beta}$. The surface covariant derivative of a vector field $\boldsymbol{q}: \omega \rightarrow \mathbb{R}^{3}$ is denoted by

$$
q_{\alpha \| \beta}=\partial_{\beta} q_{\alpha}-\Gamma_{\alpha \beta}^{\sigma} q_{\sigma}-b_{\alpha \beta} q_{3} \quad \text { and } \quad q_{3 \| \beta}=\partial_{\beta} q_{3}+b_{\beta}^{\sigma} q_{\sigma},
$$

where $\Gamma_{\alpha \beta}^{\sigma}$ are the Christoffel symbols. The covariant gradient of a scalar field $q: \omega \rightarrow \mathbb{R}$ is denoted by $\nabla_{c} q=\left(\partial_{\alpha} q\right)_{\alpha=1,2}$. In what follows, we denote by $\langle u, v\rangle$ the standard $L^{2}(\Omega)$ inner product and by $\langle u, v\rangle_{\omega}$ the $L^{2}(\Gamma)$-scalar product $\int_{w} u v \sqrt{a} d \boldsymbol{\xi}$ where $a=\operatorname{det}(\boldsymbol{A})$.

\subsection{Nonlinear Koiter shell model}

The equations for the Koiter shell model are introduced following [18]. The hypotheses are the following:

- the displacement of the structure is parallel to the normal of the reference configuration;

- the bending terms are negligible;

- the material is linear, isotropic and homogeneous.

As a consequence of these assumptions, only the membrane part of the Koiter model is considered, the shell deformation is described by the change of metric tensor $\boldsymbol{G}$ and the stress is linear in the deformation. The tensor $\boldsymbol{G}$ is a function of the displacement field $\boldsymbol{\eta}$ and reads:

$$
g_{\alpha \beta}=\frac{1}{2}\left(\boldsymbol{\eta}_{\alpha \| \beta}+\boldsymbol{\eta}_{\beta \| \alpha}\right)+a^{i j} \boldsymbol{\eta}_{i \| \alpha} \boldsymbol{\eta}_{j \| \beta}
$$


with $a^{3 \beta}=a^{\alpha 3}=0, a^{33}=1$. The constitutive law for the stress-strain relationships is expressed by means of the elastic tensor $\mathcal{E}$, whose contravariant components read:

$$
\mathcal{E}^{\alpha \beta \sigma \tau}=\frac{4 \lambda^{s} \mu^{s}}{\lambda^{s}+2 \mu^{s}} a^{\alpha \beta} a^{\sigma \tau}+2 \mu^{s} a^{\alpha \sigma} a^{\beta \tau}+2 \mu^{s} a^{\alpha \tau} a^{\beta \sigma}
$$

where $\lambda^{s}, \mu^{s}$ are the Lamé coefficients of the structure.

The equilibrium configuration for the shell (see [18] for an extensive discussion) is the stationary point of the energy functional:

$$
\psi^{\kappa}(\boldsymbol{\eta})=\frac{1}{2} \int_{\omega} \mathcal{E}^{\alpha \beta \sigma \tau} g_{\sigma \tau}(\boldsymbol{\eta}) g_{\alpha \beta}(\boldsymbol{\eta}) h_{\kappa} \sqrt{a} d \boldsymbol{\xi}-\int_{\omega} \boldsymbol{f} \cdot \boldsymbol{\eta} h_{\kappa} \sqrt{a} d \boldsymbol{\xi},
$$

where $h_{\kappa}$ is the Koiter shell thickness and $\boldsymbol{f}$ are the external forces.

Given a test field $\boldsymbol{\chi}$, defined in a suitable functional space (according to the boundary conditions of the structure), the equilibrium equations in weak form are obtained by:

$$
\Psi^{\kappa}(\boldsymbol{\eta}, \boldsymbol{\chi}):=\left\langle\delta_{\eta} \psi^{\kappa}(\boldsymbol{\eta}), \boldsymbol{\chi}\right\rangle_{\omega}=0
$$

where $\delta_{\eta}$ denotes the Fréchet derivative with respect to $\boldsymbol{\eta}$.

The form $\Psi^{\kappa}$ is specialised for the present case according to the assumptions. By using the hypothesis of a pure normal displacement, i.e. $\boldsymbol{\eta}=\eta \boldsymbol{n}$, and Eq.(1), the covariant components of the change of metric tensor $\boldsymbol{G}$ reduce to:

$$
g_{\alpha \beta}=-b_{\alpha \beta} \eta+\frac{1}{2} a^{\sigma \tau} b_{\sigma \alpha} b_{\tau \beta} \eta^{2}+\frac{1}{2} \partial_{\alpha} \eta \partial_{\beta} \eta
$$

After some algebra (see the details of the computation in Appendix A), the form reads:

$$
\begin{array}{r}
\Psi^{\kappa}(\eta, \chi):=\frac{2 E}{1-\nu^{2}} \int_{\omega}\left(c_{1} \eta-3 c_{2} \eta^{2}+2 c_{3} \eta^{3}\right) \chi-2 \nabla \chi^{T}\left(C_{1} \eta+C_{2} \eta^{2}\right) \nabla \eta+ \\
-\nabla^{T} \eta\left[\left(C_{1}+2 C_{2} \eta\right) \chi\right] \nabla \eta+\frac{1}{2}\left(\nabla \eta^{T} A^{-1} \nabla \eta\right) \nabla^{T} \chi A^{-1} \nabla \eta h_{\kappa} \sqrt{a} d \boldsymbol{\xi}- \\
\int_{\omega} \boldsymbol{f}_{n} \cdot \chi h_{\kappa} \sqrt{a} d \boldsymbol{\xi},
\end{array}
$$

where $E$ is the Young modulus of the material, $\nu$ the Poisson coefficient, the constant tensors $\left(C_{j}\right)$ and the coefficients $\left(c_{k}\right)$ are expressed as a function 
of the mean and Gauss curvatures (respectively $\rho_{1}$ and $\rho_{2}$ ) and the Poisson ratio as follows:

$$
\begin{array}{r}
c_{1}=4 \rho_{1}^{2}-2(1-\nu) \rho_{2}, \\
c_{2}=4 \rho_{1}^{3}+(\nu-3) \rho_{1} \rho_{2}, \\
c_{3}=4 \rho_{1}^{4}-4 \rho_{1}^{2} \rho_{2}+\frac{1}{2}(1+\nu) \rho_{2}^{2}, \\
C_{1}=\left[\nu \rho_{1} I+\frac{1}{2}(1-\nu) S\right] A^{-1}, \\
C_{2}=\left[\nu \rho_{1}^{2} I+\frac{1}{2}(1-\nu) S^{2}\right] A^{-1} .
\end{array}
$$

Several remarks are in order. First in the work presented in $[12,14]$ only the linear term in (3) is kept (the one multiplied by $c_{1}$ ). In this case, the shell behaves like a linear spring, whose stiffness constant depends upon the local curvatures. The non-linearities introduce two contributions: a non-linear spring and a non-linear membrane part.

\subsection{Fiber layer}

In this section, the equations for a generic fiber layer are detailed. The main hypotheses are the following:

- the energy of the shell and of the fiber layer sum up;

- from a kinematical point of view, the fibers are perfectly attached to the shell;

- the fiber is characterized by an affine stress-strain constitutive law.

The second hypothesis implies that the deformation of the fibers equals the deformation of the underlying shell structure in the direction of the fibers.

Let $\mathbf{w} \in \mathbb{T}_{\boldsymbol{x}}(\Gamma)$ be a unitary vector belonging to the tangent space of $\Gamma$ defined in the point $\boldsymbol{x} \in \Gamma$. The deformation of the fiber in the $\mathbf{w}$ direction can thus be written as:

$$
\varepsilon_{1 D}=\mathbf{w}^{T} \boldsymbol{G} \mathbf{w}=-d_{1} \eta+\frac{d_{2}}{2} \eta^{2}+\frac{1}{2} \nabla \eta^{T} P_{\mathbf{w}} \nabla \eta,
$$

where the scalar coefficients $d_{j}$ and the projector $P_{\mathbf{w}}$ are defined as $d_{1}=$ $\mathbf{w}^{T} \boldsymbol{B} \mathbf{w}, d_{2}=\mathbf{w}^{T} \boldsymbol{B} \boldsymbol{S} \mathbf{w}, P_{\mathbf{w}}=\mathbf{w} \otimes \mathbf{w}$. Note that the $d_{j}$ may be negative, 
for instance on a cylinder with outward normal. A constitutive stress-strain relationship for the fibers is assumed of the form:

$$
\sigma_{1 D}=k_{0}+k_{1} \varepsilon_{1 D}
$$

so that $k_{0}$ represents the pre-stress of the fiber and $k_{1}$ is the linear elastic coefficient.

Let $\varrho_{\mathbf{w}}$ be the fraction of the total number of fibers aligned with the direction $\mathbf{w}$ and $h_{f}$ the thickness of the fibers layer. The elastic energy of the fibers aligned in the direction $\mathbf{w}$ is expressed in the form:

$$
\psi^{\mathbf{w}}(\eta)=\frac{1}{2} \int_{\omega} \varrho_{\mathbf{w}}\left[k_{0}+k_{1} \varepsilon_{1 D}(\eta)\right] \varepsilon_{1 D}(\eta) h_{f} \sqrt{a} d \boldsymbol{\xi}+\int_{\omega} r_{\mathbf{w}} h_{f} \sqrt{a} d \boldsymbol{\xi}
$$

where $r_{\mathbf{w}}$ represents the potential energy of a force acting on the fibers aligned with the direction $\mathbf{w}$.

The equilibrium equations are introduced in weak form, as the scalar product of the Fréchet derivative of the energy with a test function:

$$
\begin{aligned}
& \Psi^{\mathbf{w}}(\eta, \chi)=\int_{\omega} \varrho_{\mathbf{w}} \nabla \chi^{T}\left[k_{0}+k_{1}\left(-d_{1}+\frac{d_{2}}{2} \eta^{2}\right)+\frac{k_{1}}{4} W\right] P_{\mathbf{w}} \nabla \eta+ \\
& \varrho_{\mathbf{w}}\left[k_{0}\left(-d_{1}+d_{2} \eta\right)+k_{1}\left(-d_{1}^{2} \eta-\frac{3 d_{1} d_{2}}{2} \eta^{2}+\frac{d_{2}^{2}}{2} \eta^{3}\right)+\frac{k_{1}}{2}\left(-d_{1}+d_{2} \eta\right) W\right] \chi+ \\
& \left(\delta_{\eta} r_{\mathbf{w}}\right) \chi h_{f} \sqrt{a} d \boldsymbol{\xi}
\end{aligned}
$$

where $W=\left(\nabla \eta^{T} P_{\mathbf{w}} \nabla \eta\right)$. Remark that the contribution of the first line is of the membrane type, whereas the second line contains algebraic terms in the test function and hence it renders a non-linear spring-like behavior.

When $\eta=0$, the reference configuration is the equilibrium configuration only if the stress exerted by the fibers due to their pre-stress is balanced by the underlying shell. This, in weak form, can be written as:

$$
\int_{\omega}\left(-\varrho_{\mathbf{w}} k_{0} d_{1}+\delta_{\eta} r_{\mathbf{w}}\right) \chi h_{f} \sqrt{a} d \boldsymbol{\xi}=0
$$

that holds for arbitrary test functions $\chi$, hence

$$
r_{\mathbf{w}}=\varrho_{\mathbf{w}} k_{0} d_{1} \eta
$$


To conclude this section, let us remark that, in general, the fibers are not parallel to only one direction. Consider two linearly independent unitary vectors $\mathbf{w}, \mathbf{v} \in \mathbb{T}_{\boldsymbol{x}}(\Gamma)$ and the associated fiber densities $\varrho_{\mathbf{w}}, \varrho_{\mathbf{v}}$ defined in each point of $\Gamma$. In such a case we can simply sum the two associated energies $\psi^{\mathbf{w}}$ and $\psi^{\mathbf{v}}$.

\subsection{Equations for the structure dynamics}

The equations for the structure dynamics are obtained by adding the inertia terms to the elastic contributions highlighted in the previous sections. In particular the dynamics equations in weak form can be written as:

$$
\int_{\omega} \rho^{s} h^{s}\left(\partial_{t t}^{2} \eta\right) \chi \sqrt{a} d \boldsymbol{\xi}+\Psi^{\kappa}(\eta, \chi)+\Psi^{\mathbf{w}}(\eta, \chi)+\Psi^{\mathbf{v}}(\eta, \chi)=0,
$$

where $\Psi^{\kappa}$, defined in Eq.(3), represents the contribution of the shell and $\Psi^{\mathbf{w}}, \Psi^{\mathbf{v}}$, defined in Eq.(6), represent the contribution of the fibers aligned in the directions $\mathbf{w}$ and $\mathbf{v}$ respectively. The total thickness of the structure is denoted by $h^{s}$ and its density is denoted by $\rho^{s}$.

In the following, for the sake of simplicity, the overall contribution of the structure will be denoted as:

$$
\Psi^{s}=\Psi^{\kappa}+\Psi^{\mathrm{w}}+\Psi^{\mathbf{v}}
$$

\section{Fluid-structure coupling}

The fluid is governed by the incompressible Navier-Stokes equations:

$$
\begin{array}{ll}
\rho^{f}\left(\partial_{t} \boldsymbol{u}+\boldsymbol{u} \cdot \nabla \boldsymbol{u}\right)=\nabla \cdot \boldsymbol{\sigma}^{f} & \text { in } \Omega_{t} \\
\nabla \cdot \boldsymbol{u}=0 & \text { in } \Omega_{t}
\end{array}
$$

where $\boldsymbol{u}$ is the velocity, $\rho^{f}$ is the fluid density and $\boldsymbol{\sigma}^{f}=\mu^{f}\left(\nabla \boldsymbol{u}+(\nabla \boldsymbol{u})^{T}\right)-p \boldsymbol{I}$ is the fluid stress tensor, where $\mu^{f}$ is the dynamic viscosity and $p$ is the pressure. The domain $\Omega_{t}$ is, in general, time-dependent, since the wall is an elastic structure which is moving because of the interactions with the fluid. We denote by $\Omega$ a fixed reference domain. We normalize both the equations for the structure and the fluid by $\rho^{f}$ and we introduce the kinematic viscosity $\nu^{f}=\frac{\mu^{f}}{\rho^{f}}$. The quantities $E, k_{0}, k_{1}, f^{s}, \rho^{s}$ and $p$ are also divided by $\rho^{f}$, but for the sake of simplicity their notation is not changed.

The boundary $\partial \Omega_{t}$ is subdivided into two subsets $\Gamma_{t}$, the interface between the fluid and the structure, and $\Sigma_{t}$, representing the artificial boundaries of 
the domain where inlet and outlet boundary conditions are enforced on the normal component of the stress tensor.

Two conditions have to be satisfied on the fluid-structure interface $\Gamma_{t}$ : the continuity of the velocity and the stress. Since the structure displacement is assumed to be parallel to the normal direction, it holds, for $\boldsymbol{x} \in \Gamma, \boldsymbol{u}(\boldsymbol{I}-\boldsymbol{n} \otimes$ $\boldsymbol{n})\left.\right|_{\boldsymbol{x}+\eta(\boldsymbol{x}) \boldsymbol{n}(\boldsymbol{x})}=\mathbf{0}$ and $\left.\boldsymbol{u} \cdot \boldsymbol{n}\right|_{\boldsymbol{x}+\eta(\boldsymbol{x}) \boldsymbol{n}(\boldsymbol{x})}=\partial_{t} \eta$. The continuity of the normal component of the normal stress gives $\left.\boldsymbol{\sigma}_{\boldsymbol{n} \boldsymbol{n}}^{f}\right|_{\boldsymbol{x}+\eta(\boldsymbol{x}) \boldsymbol{n}(\boldsymbol{x})}=-f^{s}-p^{r e f}$, where $p^{\text {ref }}$ denotes a given external pressure acting on the structure.

As stated in the introduction, the aim of the present work is to set up a simplified fluid-structure interaction model, that can provide solutions at a moderate computational cost. To this end, the structure equations are treated as a boundary condition for the fluid problem and the problem is discretized on a fixed mesh. In order to render the motion of the wall, a transpiration approach is adopted. A zero-th order transpiration was investigated in $[14,19,15]$, and proves to be satisfactory to study the propagation of pressure waves. However, in view of the application that motivated this work [17] (hemodynamic autoregulation), it is important to compute the flow variation induced by the wall dynamics. Thus, a first order transpiration condition is considered.

We denote by $\boldsymbol{y} \in \Gamma_{t}$ a point on the actual boundary and by $\boldsymbol{x} \in \Gamma$ the corresponding point on the reference configuration. The mapping $\boldsymbol{y}=\Phi(\boldsymbol{x})$ is written as $\boldsymbol{y}=\boldsymbol{y}(\boldsymbol{x})=\boldsymbol{\eta}(\boldsymbol{x})+\boldsymbol{x}$. The displacement is assumed to be given by $\boldsymbol{\eta}(\boldsymbol{x})=\eta(\boldsymbol{x}) \boldsymbol{n}(\boldsymbol{x})$, where $\boldsymbol{n}(\boldsymbol{x})$ is the outward normal to the reference domain at the point $\boldsymbol{x}$. An additional assumption is made: the normal is supposed to remain the same during the evolution, that is $\boldsymbol{n}(\boldsymbol{x})=\boldsymbol{n}(\boldsymbol{y})$. This assumption, which was also used in $[12,14]$, can be obtained by assuming small deformations since the difference $|\boldsymbol{n}(\boldsymbol{x})-\boldsymbol{n}(\boldsymbol{y})|$ is of order one in $\nabla \eta$. The first order transpiration conditions are obtained through a first order Taylor expansion around the reference configuration $\eta=0$ :

$$
\begin{gathered}
\boldsymbol{u}(\boldsymbol{y})=\boldsymbol{u}(\boldsymbol{x})+\nabla \boldsymbol{u}(\boldsymbol{x})(\boldsymbol{y}-\boldsymbol{x})+O\left(\|\boldsymbol{y}-\boldsymbol{x}\|^{2}\right), \\
\boldsymbol{u}(\boldsymbol{y})=\boldsymbol{u}(\boldsymbol{x})+(\eta \nabla \boldsymbol{u n})(\boldsymbol{x})+O\left(\eta^{2}\right),
\end{gathered}
$$

where the gradient is taken with respect to the $\boldsymbol{x}$ coordinate. The tangential component of the velocity is computed by multiplying Eq.(8) by $(\boldsymbol{I}-\boldsymbol{n} \otimes \boldsymbol{n})$ :

$$
(\boldsymbol{I}-\boldsymbol{n} \otimes \boldsymbol{n}) \boldsymbol{u}(\boldsymbol{y})=(\boldsymbol{I}-\boldsymbol{n} \otimes \boldsymbol{n})(\boldsymbol{u}(\boldsymbol{x})+\eta \nabla \boldsymbol{u}(\boldsymbol{x}) \boldsymbol{n}(\boldsymbol{x}))+O\left(\eta^{2}\right) .
$$


Using the no-slip boundary conditions and neglecting the high-order terms:

$$
(\boldsymbol{I}-\boldsymbol{n} \otimes \boldsymbol{n}) \boldsymbol{u}(\boldsymbol{x})=-\eta(\boldsymbol{I}-\boldsymbol{n} \otimes \boldsymbol{n})(\nabla \boldsymbol{u}(\boldsymbol{x}) \boldsymbol{n}(\boldsymbol{x})) \quad \text { on } \Gamma .
$$

From Eq.(8), the normal component of the velocity can be written as

$$
\boldsymbol{n} \otimes \boldsymbol{n u}(\boldsymbol{y})=\boldsymbol{n} \otimes \boldsymbol{n}(\boldsymbol{u}(\boldsymbol{x})+\eta \nabla \boldsymbol{u}(\boldsymbol{x}) \boldsymbol{n}(\boldsymbol{x}))+O\left(\eta^{2}\right) .
$$

Neglecting high order terms, the continuity of the normal velocity gives:

$$
\partial_{t} \eta=\boldsymbol{u} \cdot \boldsymbol{n}+\eta \nabla \boldsymbol{u n} \cdot \boldsymbol{n} .
$$

We make the additional simplifying assumption that the viscous part of $\left.\boldsymbol{\sigma}_{\boldsymbol{n} \boldsymbol{n}}^{f}\right|_{\boldsymbol{x}+\eta(\boldsymbol{x}) \boldsymbol{n}(\boldsymbol{x})}$ is negligible compared to the pressure, i.e, $\left.\boldsymbol{\sigma}_{\boldsymbol{n} \boldsymbol{n}}^{f}\right|_{\boldsymbol{x}+\eta(\boldsymbol{x}) \boldsymbol{n}(\boldsymbol{x})}=$ $-\left.p\right|_{\boldsymbol{x}+\eta(\boldsymbol{x}) \boldsymbol{n}(\boldsymbol{x})}$. Then, the value of $\left.\boldsymbol{\sigma}_{\boldsymbol{n} \boldsymbol{n}}^{f}\right|_{\boldsymbol{x}+\eta(\boldsymbol{x}) \boldsymbol{n}(\boldsymbol{x})}$ is approximated by using a first order Taylor expansion on $p$

$$
\left.\boldsymbol{\sigma}_{\boldsymbol{n} \boldsymbol{n}}^{f}\right|_{\boldsymbol{x}+\eta(\boldsymbol{x}) \boldsymbol{n}(\boldsymbol{x})}=-p-\eta \nabla p \cdot \boldsymbol{n}
$$

The equations for the coupled system are written in weak form, on a fixed reference frame. Let $\boldsymbol{v}, q, \chi, \boldsymbol{w}$ be test functions defined in suitable functional spaces according to the boundary conditions of the problem. In particular let $(\boldsymbol{u}, \boldsymbol{v}) \in V \subset H^{2}(\Omega)$, let $(p, q) \in M \subset H^{1}(\Omega)$ and $(\eta, \chi) \in H^{1}(\Gamma)$, $\boldsymbol{w} \in H^{1}(\mathbb{T}(\Gamma))$. Then:

$$
\begin{cases}\left\langle\partial_{t} \boldsymbol{u}, \boldsymbol{v}\right\rangle+c(\boldsymbol{u} ; \boldsymbol{u}, \boldsymbol{v})+a(\boldsymbol{u}, \boldsymbol{v})+b(p, \boldsymbol{v})=0 & \text { in } \Omega, t>0 \\ \langle\nabla \cdot \boldsymbol{u}, q\rangle=0 & \text { in } \Omega, t>0 \\ \rho_{s} h_{s}\left\langle\partial_{t t}^{2} \eta, \chi\right\rangle_{\omega}+\Psi^{s}(\eta, \chi)+\left\langle p^{r e f}, \chi\right\rangle_{\omega}=\langle p+\eta \nabla p \cdot \boldsymbol{n}, \chi\rangle_{\omega} & \text { on } \Gamma \\ \left\langle\partial_{t} \eta, \chi\right\rangle_{\omega}=\langle\boldsymbol{u} \cdot \boldsymbol{n}+\eta \nabla \boldsymbol{u n} \cdot \boldsymbol{n}, \chi\rangle_{\omega} & \text { on } \Gamma \\ \langle(\boldsymbol{I}-\boldsymbol{n} \otimes \boldsymbol{n})(\boldsymbol{u}+\eta \nabla \boldsymbol{u n}), \boldsymbol{w}\rangle_{\omega}=0 & \text { on } \Gamma .\end{cases}
$$

The forms $a, b, c$ read:

$$
\begin{array}{ll}
a: \boldsymbol{V} \times \boldsymbol{V} \rightarrow \mathbb{R}, \quad a(\boldsymbol{u}, \boldsymbol{v})=\nu^{f}\left(\nabla \boldsymbol{u}+\nabla \boldsymbol{u}^{T}, \nabla \boldsymbol{v}\right)_{\Omega} & \forall(\boldsymbol{u}, \boldsymbol{v}) \in \boldsymbol{V} \times \boldsymbol{V} \\
b: M \times \boldsymbol{V} \rightarrow \mathbb{R}, \quad b(p, \boldsymbol{v})=-(p, \nabla \cdot \boldsymbol{v})_{\Omega} & \forall(p, \boldsymbol{v}) \in M \times \boldsymbol{V} \\
c(\boldsymbol{w}): \boldsymbol{V} \times \boldsymbol{V} \rightarrow \mathbb{R}, \quad c(\boldsymbol{w} ; \boldsymbol{u}, \boldsymbol{v})=(\boldsymbol{w} \cdot \nabla \boldsymbol{u}, \boldsymbol{v})_{\Omega} & \forall(\boldsymbol{u}, \boldsymbol{v}) \in \boldsymbol{V} \times \boldsymbol{V}
\end{array}
$$




\section{Numerical discretization}

\subsection{Time discretization}

The approximation of the generic quantity $f$ at time $t_{k}=k \Delta t$ is denoted by $f^{k}$. The following notation is introduced: $\nabla_{\boldsymbol{n}} f=\nabla f \cdot \boldsymbol{n}, \nabla_{\boldsymbol{n} \boldsymbol{n}} \boldsymbol{f}=\nabla \boldsymbol{f} \boldsymbol{n} \cdot \boldsymbol{n}$, $f_{\boldsymbol{n}}=\boldsymbol{f} \cdot \boldsymbol{n}$.

An implicit-explicit time discretization is adopted in order to avoid the resolution of non-linear problems. As regards the tangential velocity boundary condition, the Taylor contribution accounting for the motion of the wall is taken at the current time step:

$$
\left\langle(\boldsymbol{I}-\boldsymbol{n} \otimes \boldsymbol{n}) \boldsymbol{u}^{k+1}, \boldsymbol{w}\right\rangle_{\omega}=-\left\langle\eta^{k}(\boldsymbol{I}-\boldsymbol{n} \otimes \boldsymbol{n}) \nabla \boldsymbol{u}^{k} \boldsymbol{n}, \boldsymbol{w}\right\rangle_{\omega} \text { on } \Gamma .
$$

The normal component of the velocity, which is directly related to the wall displacement $\eta$ is discretized by adopting a similar strategy:

$$
\left\langle\eta^{k+1}, \chi\right\rangle_{\omega}=\left\langle\left(1+\Delta t \nabla_{\boldsymbol{n} \boldsymbol{n}} \boldsymbol{u}^{k}\right) \eta^{k}, \chi\right\rangle_{\omega}+\Delta t\left\langle u_{\boldsymbol{n}}^{k+1}, \chi\right\rangle_{\omega} .
$$

The structure equation is discretized as follows:

$\rho_{s} h_{s}\left\langle\frac{\eta^{k+1}-2 \eta^{k}+\eta^{k-1}}{\Delta t^{2}}, \chi\right\rangle_{\omega}+\Psi^{s}\left(\eta^{k+1, k}, \chi\right)+\left\langle p^{r e f}, \chi\right\rangle_{\omega}=\left\langle p^{k+1}+\eta^{k} \nabla_{\boldsymbol{n}} p^{k}, \chi\right\rangle_{\omega}$,

where $\Psi^{s}$ is the energy of the structure defined in Eq. (7). The non-linear terms in $\Psi^{s}$ are treated in a semi-implicit way detailed in Appendix B (from equations (B.1) to (B.3)). After linearization $\Psi^{s}$ is replaced by the sum of a bilinear form of $\eta^{k+1}$ and $\chi$ and a linear functional of $\chi$. In order to eliminate the current displacement from the the bilinear form, every istance of $\eta^{k+1}$ is replaced by Eq.(11) to obtain:

$$
\Psi^{s}\left(\eta^{k+1, k}, \chi\right)=\Phi^{k+1}\left(u_{n}^{k+1}, \chi\right)+\phi^{k}(\chi)
$$

The bilinear form $\Psi^{s}\left(\eta^{k+1, k}, \chi\right)$ is now divided into two contributions: the first one $\Phi^{k+1}\left(u_{n}^{k+1}, \chi\right)$ is a bilinear form that depends on the current velocity and the second one $\phi^{k}(\chi)$ is a linear functional of $\chi$ where old quantities appear as parameters (see Eq. (B.4) for detailed expressions). By injecting Eq.(13) into Eq.(12) and by collecting all the force terms in one functional, the following is obtained:

$$
\frac{\rho^{s} h^{s}}{\Delta t}\left\langle u_{\boldsymbol{n}}^{k+1}, \chi\right\rangle_{\omega}+\Phi^{k+1}\left(u_{\boldsymbol{n}}^{k+1}, \chi\right)-\left\langle p^{k+1}, \chi\right\rangle_{\omega}=\mathcal{F}\left(\chi ; \eta^{k}, p^{k}, u_{\boldsymbol{n}}^{k}\right)
$$


The details of the expression for $\mathcal{F}$ are reported in Eq. (B.5). Remark that the left-hand side is now made up of the unknowns of the fluid problem, and the right-hand side is computed by using the values of the variables at the previous time step. In this way, the motion of the structure has been implicitly embedded as a boundary condition for the fluid problem. In addition, as the acceleration of the structure is treated implicitly, possible numerical instabilities due to the added-mass effect [20] are avoided.

The linear part of the Navier-Stokes equations is discretized by an implicit Euler scheme, and the convective term in a semi-implicit way: $c\left(\boldsymbol{u}^{k} ; \boldsymbol{u}^{k+1}, \boldsymbol{v}\right)=$ $\left\langle\boldsymbol{u}^{k} \cdot \nabla \boldsymbol{u}^{k+1}, \boldsymbol{v}\right\rangle$.

Some comments on the stability of the scheme are in order. In the work by Nobile and Vergara [12], stability was proven for a similar model where the fluid problem was solved on a moving domain and a linear Koiter model was embedded into the fluid boundary conditions. In the work by Pironneau [14] a complete mathematical analysis of a similar method was done on a fixed domain where the coupling was defined by a zero order transpiration. The proof of stability of the formulation proposed in the present work seems to be more complicated because of the first order transpiration that results in a non-linear mixed boundary condition on the surface $\Gamma$ for the continuous problem.

\subsection{Application of the boundary conditions}

Eq.(9) is discretized using P1-P1 finite elements with an SUPG stabilization. In this section, several remarks on the imposition of the boundary conditions for the system are presented.

The boundary conditions are separated into a tangential boundary condition of Dirichlet type for the velocity and a generalised Robin boundary condition in the normal direction. In order to impose the conditions via penalization and to avoid cancellation errors, a unique vector-type boundary condition is written. Let $\boldsymbol{z}=\boldsymbol{w}+\chi \boldsymbol{n}$ be a test function, with $\boldsymbol{w} \cdot \boldsymbol{n}=0$. It holds:

$$
-\left\langle p^{k+1} \boldsymbol{n}, \boldsymbol{z}\right\rangle_{\omega}+\ell\left(\boldsymbol{u}^{k+1}, \boldsymbol{z}\right)=\mathcal{R}(\boldsymbol{z}),
$$

where $\ell$ is a bilinear form and $\mathcal{R}$ is a linear functional, deduced from Eq.(14) and Eq.(10) (see Appendix B).

The implementation of this boundary condition has to be done carefully when working with real geometries. In particular, we observed that with normals constant per element, spurious contributions could appear on the 
tangential velocity (see Remark below). It is therefore desirable to reinterpolate the normals on the P1 finite elements nodes.

Remark 1. Let us explain the trouble that may come from a piecewise-P0 approximation of the normal when a penalization method on the weak form is adopted. Consider, for instance, the case of a Dirichlet boundary condition $\boldsymbol{u}=(\boldsymbol{I}-\boldsymbol{n} \otimes \boldsymbol{n}) \boldsymbol{g}$, where $\boldsymbol{g}$ is a generic vector defined on $\Gamma$. When this condition is imposed via penalization the weak formulation reads:

$$
\int_{\Gamma}(\boldsymbol{u}-\boldsymbol{T g}) \cdot \boldsymbol{w} \approx 0
$$

where $\boldsymbol{T}=(\boldsymbol{I}-\boldsymbol{n} \otimes \boldsymbol{n})$ is the projector in the tangent space, and " $\approx 0$ " means "of the order of the inverse of the penalization parameter". If a piecewiseP0 approximation to the normal field is used, the projector operator $\boldsymbol{T}^{e l}$ is defined element-wise and is discontinuous. The $k$-th component of this equation is written by introducing the basis functions used to discretize the problem, namely $\varphi$, providing:

$$
\int_{\Gamma}\left(u_{k, i} \varphi_{i}-\left(T_{k r}^{e l} g_{r}\right)_{i} \varphi_{i}\right) w_{k, j} \varphi_{j} \approx 0
$$

where $\left(T_{k, j}^{e l} g_{j}\right)_{i}=\int_{\Gamma} T_{k, r}^{e l} g_{r} \phi_{i}$. This equation leads to $u_{k, i} \approx\left(T_{k, r}^{e l} g_{r}\right)_{i}$, and hence it does not guarantee that $n_{s}^{e l} n_{k}^{e l} u_{k, i} \approx 0$, for $s=1,2,3$. On the other hand, if the piecewise-P1 normal and the corresponding operator $\boldsymbol{T}$ are used, the quantity $\boldsymbol{T} \boldsymbol{g}$ is computed for each node and the following identity holds:

$$
\int_{\Gamma}\left(u_{k, i} \varphi_{i}-T_{k r, i} g_{r, i} \varphi_{i}\right) \varphi_{j} \approx 0, \quad l=1 \ldots 3
$$

which leads to $u_{k, i} \approx T_{k r, i} g_{r, i}$. The velocity satisfies the condition $n_{s} n_{k} u_{k, i}=$ 0 , for $s=1,2,3$. This is particularly relevant for the accuracy in the computation of the normal component of the velocity and thus of the displacement field. $\diamond$

\section{Numerical testcases}

In this section, three numerical experiments are presented where the different features of the proposed method are tested.

In the first test case, a pressure wave in a circular cylinder is simulated, in a similar setting to that in [12]. In the second numerical experiment, a similar 


\begin{tabular}{lllll}
\hline & Koiter layer & \multicolumn{3}{l}{ Fibers Layer } \\
& $E\left[\mathrm{dyn} / \mathrm{cm}^{2}\right]$ & $h_{\kappa}[\mathrm{cm}]$ & $k_{0}\left[\mathrm{dyn} / \mathrm{cm}^{2}\right]$ & $h_{f}[\mathrm{~cm}]$ \\
\hline Without fibers & $7.5 \cdot 10^{5}$ & 0.1 & 0 & 0 \\
With fibers & $3.75 \cdot 10^{5}$ & 0.1 & $2.78 \cdot 10^{5}$ & 0.1 \\
\hline
\end{tabular}

Table 1: Structure parameters for the two simulations.

problem is solved in a different geometry, characterized by the presence of a flat region. Finally, the proposed method is applied to an image-based geometry describing a part of the retinal arteriolar network.

\subsection{Pressure wave in a cylinder}

In this test case, the Stokes equations are solved in a cylindrical domain $(L=6 \mathrm{~cm}, R=0.5 \mathrm{~cm})$. Two different configurations are compared, one with fibers and one without. The parameters are reported in Tab.1. The values for the Young modulus and for the fibers pre-stress are chosen such that, after linearization, the spring coefficient $\left(\frac{h^{k} E}{\left(1-\nu^{s^{2}}\right) R^{2}}+\frac{h_{f} k_{0} \varrho_{\mathbf{w}}}{R^{2}}\right)$ coincides for the two configurations. The remaining structure parameters are: $\rho^{s}=1$ $\mathrm{g} / \mathrm{cm}^{3}, h_{s}=h_{\kappa}+h_{f}$ and $\nu^{s}=0.5$. For the sake of simplicity, we set $k_{1}=0$. Fibers are assumed to be aligned with the principal directions of curvature: $\mathbf{w}$ is the circumferential direction and $\mathbf{v}$ the longitudinal direction. The fibers' fraction is chosen such that fibers are mostly aligned with the circumferential direction $\left(\varrho_{\mathbf{w}}=0.9, \varrho_{\mathbf{v}}=0.1\right)$.

For such a simple geometry, the fibers' directions and curvatures are known analytically. However, in view of applying the method to realistic geometries, where this information is in general not available, we assume that the directions and the curvatures are unknown, and we estimate them numerically from the computational mesh.

Fig.1 presents curvature estimations. On the left, the Gaussian curvature (analytically equal to zero) and the mean curvature (analytically equal to one) are plotted against the longitudinal coordinate of the cylinder. Notice the presence of numerical oscillations in the computation of the curvature. This problem may have negative effects on other simplified FSI models, as observed in [13]. In the proposed model, it is partially overcome by the addition of the fiber layer as shown below. In the right panel of Fig.1, the estimated principal curvature directions are shown.

The fluid parameters are: $\rho^{f}=1 \mathrm{~g} / \mathrm{cm}^{3}$ and $\mu^{f}=0.035 \mathrm{~cm}^{2} / \mathrm{s}$. The 

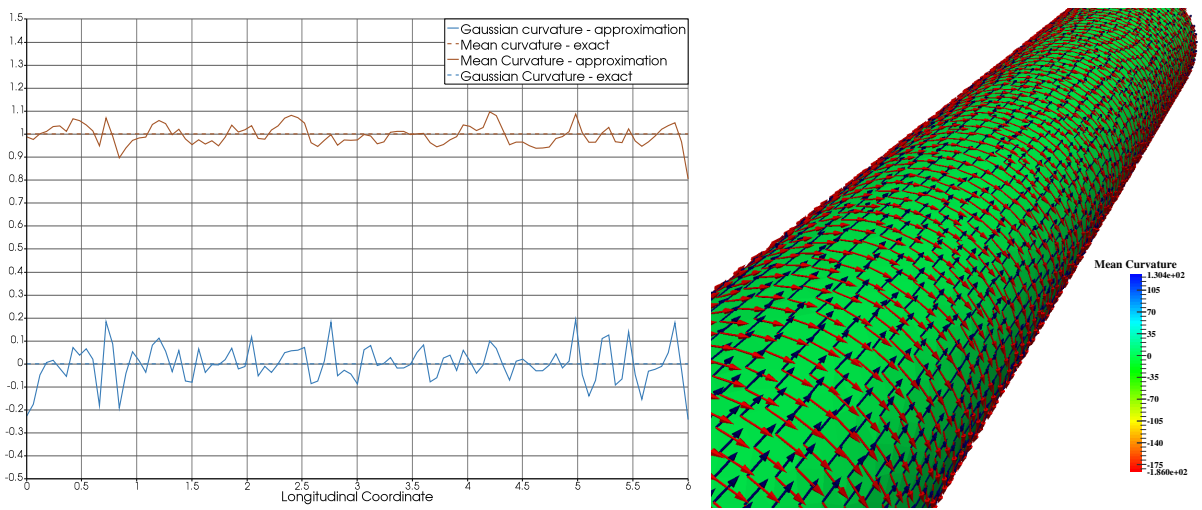

Figure 1: On the left panel the mean (brown) and the Gaussian (blue) curvature estimated from the computational mesh are compared to the exact values: one and zero, respectively. On the right panel the principal directions of curvature are depicted: red arrows represent the local direction of maximum curvature, the blue ones refer to the minimum. On the surface the mean curvature is displayed.

boundary conditions at the inlet and at the outlet are $\boldsymbol{\sigma}(\boldsymbol{u}, p) \boldsymbol{n}=-\overline{\boldsymbol{n}} \boldsymbol{n}$. A pressure equal to zero is assigned at the outlet $(\bar{p}=0)$ and at the inlet

$$
\bar{p}= \begin{cases}5000 \mathrm{dyn} / \mathrm{cm}^{2} & t \leq 0.005 s \\ 0 & t>0.005 s\end{cases}
$$

In Figures 2 and 3 the displacement is shown against the longitudinal coordinate, for two different time instants: $t=0.004$ and $t=0.012$, respectively. First, there is a difference between the non-linear model and its linear version, both with and without the fiber layer. The pressure wave amplitude is lower for the non-linear model, and the wave velocity is higher. This results from the fact that the non-linear model is characterized by a greater stiffness (due to the non-linear spring contribution).

By comparing the models with and without fibers, it is interesting to notice the regularizing effect of the fibers. The difference in the peak amplitude and in the wave velocity is due to the difference in inertia and stiffness.

\subsection{Pressure wave in a flat cylinder}

The effect of adding a fiber layer to the structure model is even more visible in presence of flat regions in the surface. In realistic geometries, a locally flat region may occur for several reasons including a lack of precision 


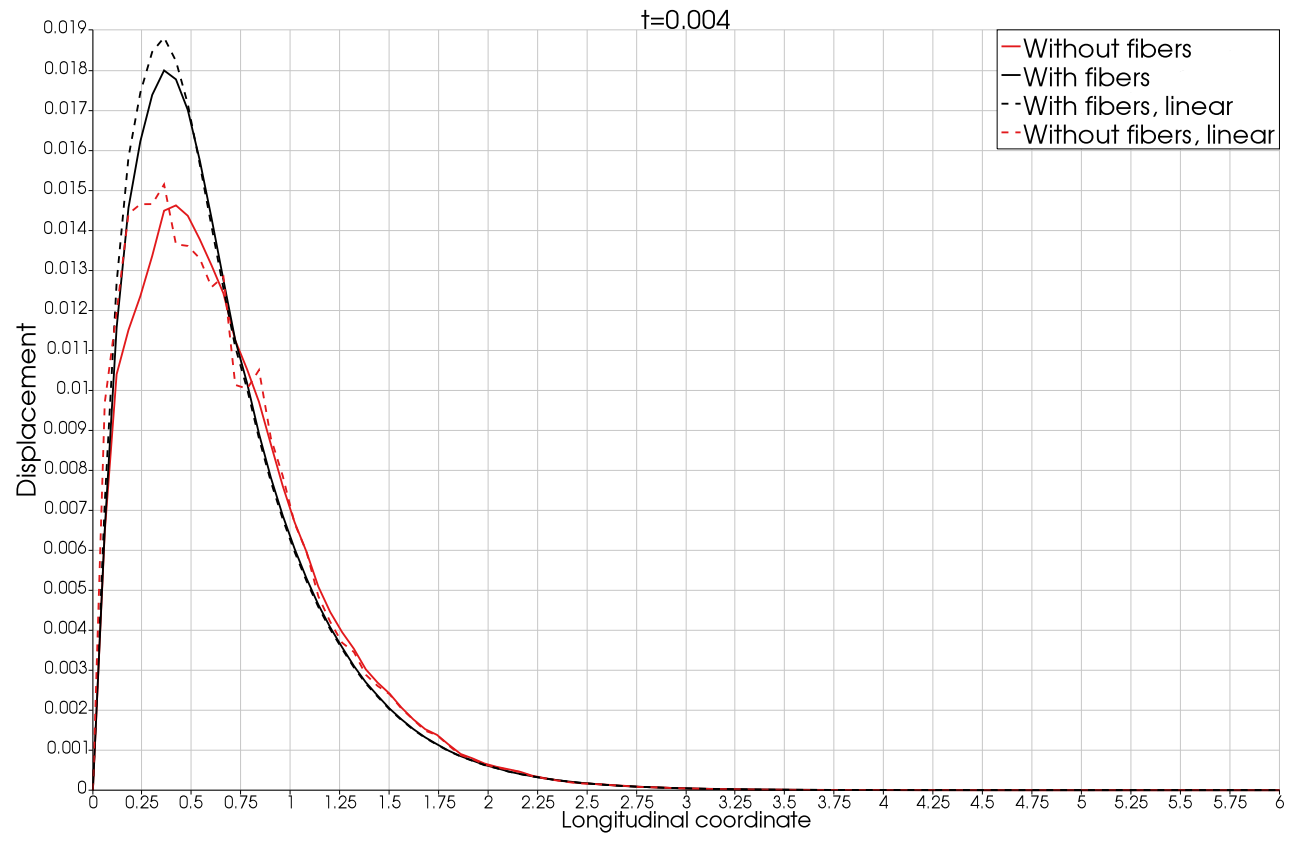

Figure 2: Displacement in the longitudinal direction for different structure parameters (see Table 1) for $t=0.004$. Red lines refer to the case without fibers, while black ones to the case with fibers. Solid lines refer to the full model and dashed lines to the corresponding linear version. 


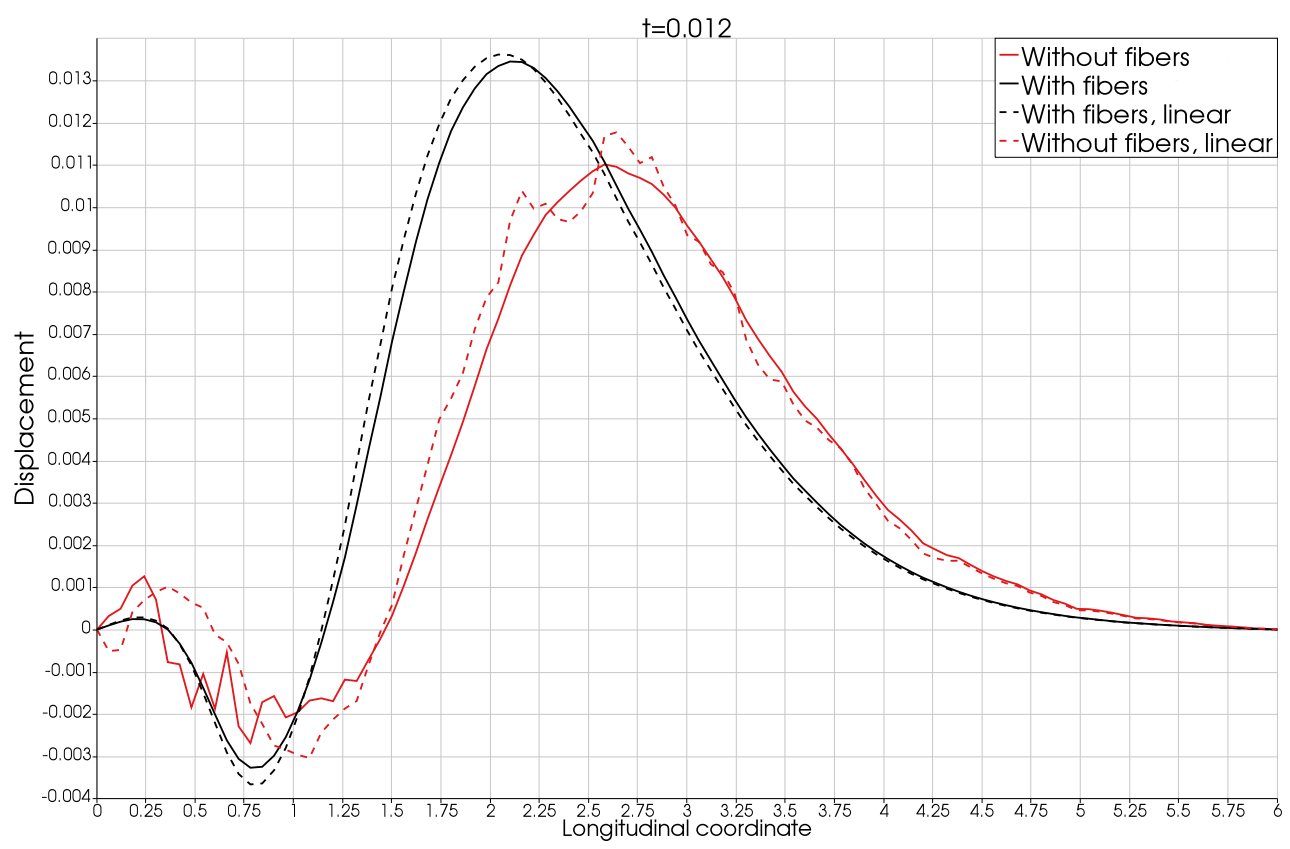

Figure 3: Displacement in the longitudinal direction for different structure parameters (see Table 1) for $t=0.012$. Red lines refer to the case without fibers, while black ones to the case with fibers. Solid lines refer to the full model and dashed lines to the corresponding linear version.

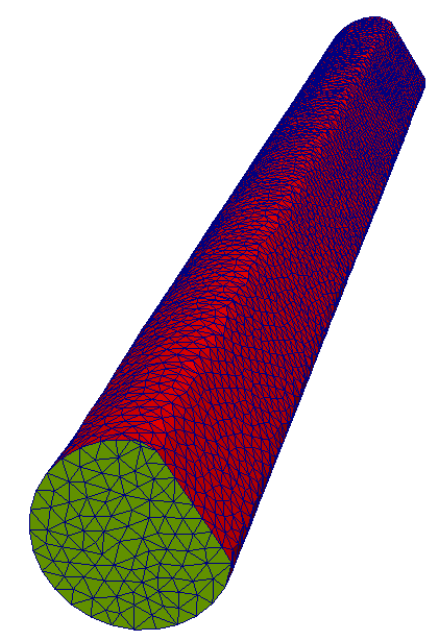

Figure 4: The domain of the second test case. One side of the cylinder is flat, i.e. all the curvatures vanish. 


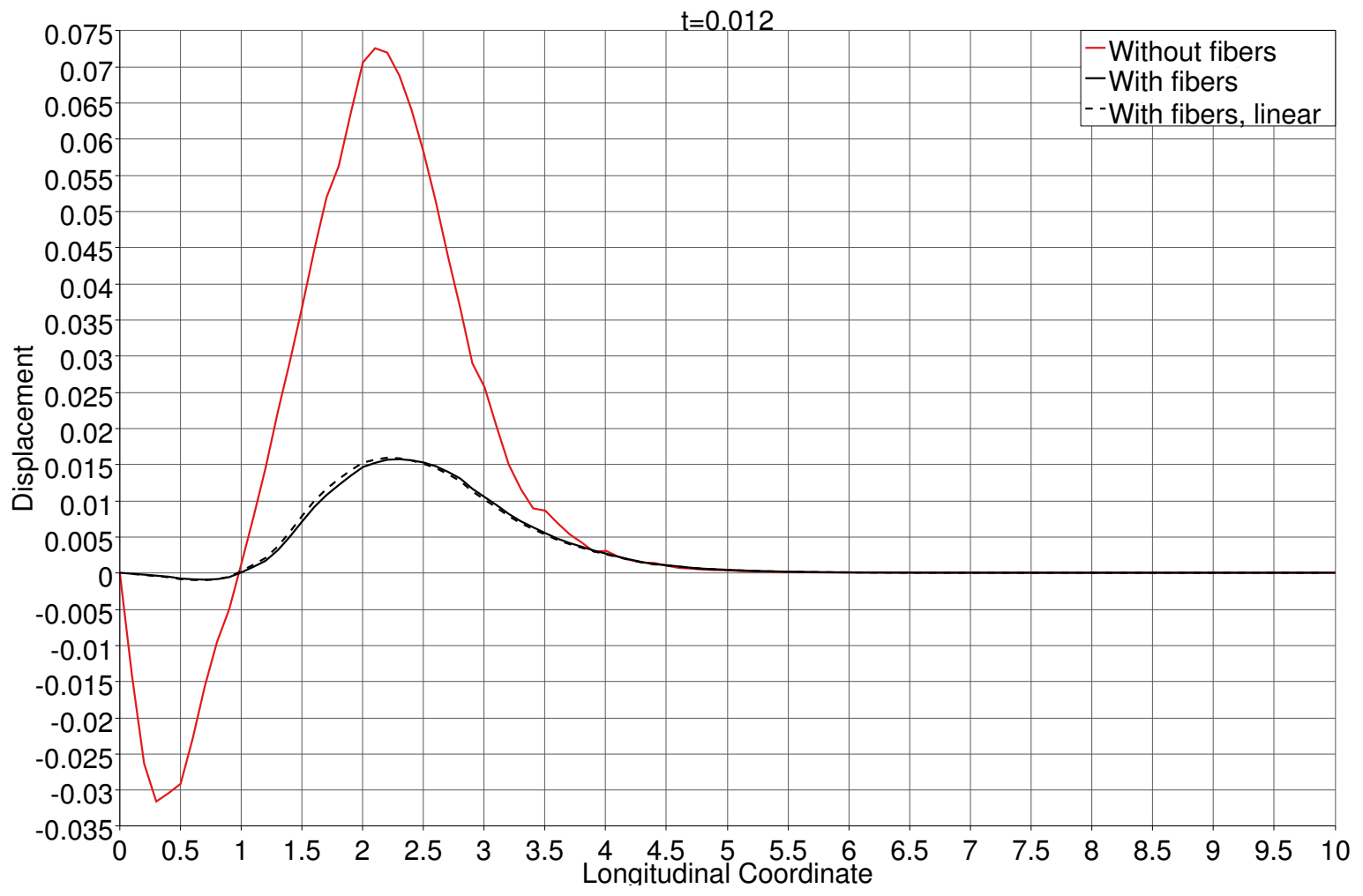

Figure 5: Displacement in the flat region. Comparison between the model with fibers (black dashed line linear, black solid line full-model) and the model without (red solid line).

in the segmentation process. This is why the fluid-structure interaction in a flattened cylinder (see Figure 4) is investigated.

For this geometrical setting the inertial-algebraic model proposed in [12] cannot be used. The main reason is that this model reduces to a structure that behaves pointwise like a spring, whose stiffness constant depends on the curvatures. When both the curvatures vanish, the spring coefficient is zero. Therefore, the displacement becomes very large, leading to unphysical solutions. Adding the non-linear part of the Koiter shell model mitigates this behavior.

In Fig. 5 the displacement is shown in the longitudinal direction in the center of the flat region for $t=0.012$. Notice that, in the flat region, the structure without fibers is characterized by a significantly large displacement. This is due to the fact that, in this region, the structure behaves like a 


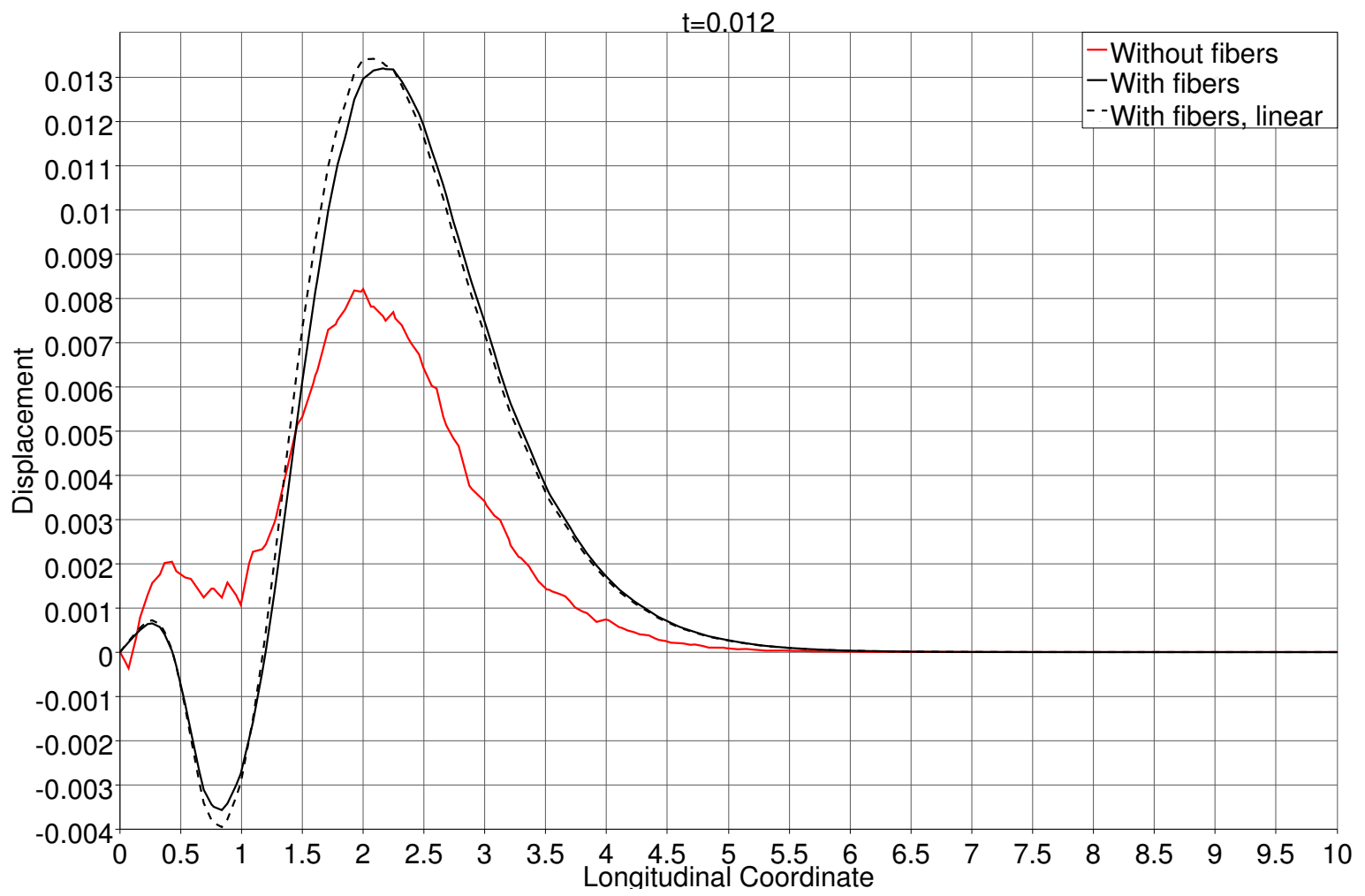

Figure 6: Displacement in the rounded region. Comparison between the model with fibers (black dashed line linear, black solid line full-model) and the model without (red solid line).

membrane, whose stiffness is low. In Fig.6 the same curves are reported for the displacement in the non-flat region. In this portion, the behavior of the structure is similar to that found in the first test case. 


\section{Application}

In the last test case the model is applied to a realistic geometry. The network represents the inferior temporal arteriole network in the human retina. The original image was taken in the Drive dataset [21]. The vasculature has been segmented by applying the imaging methods presented in $[22,23]$. The $2 \mathrm{D}$ data have then been expanded into a 3D-network by assuming a circular section and projecting the results onto a sphere representing the eye. The typical diameter of this network varies between $70 \mu \mathrm{m}$ and $160 \mu \mathrm{m}$.

Several physical parameters appear in the equations of the structure and in that of the fluid. The parameters are chosen to be comparable to those found in $[16,24,25]$ and they are a realistic and representative set of parameters for retinal arterioles. The parameters used for the arteriole wall are $\rho_{s}=1 \mathrm{~g} / \mathrm{cm}^{3}, E=0.05 M P a, \nu=0.5, h_{\kappa}=5 \mu m, h_{f}=20 \mu m, k_{0}=0.4 M P a$, $k_{1}=0, \varrho_{\mathbf{w}}=\varrho_{\mathbf{v}}=0.5$. For the sake of simplicity, the blood is assumed to be Newtonian, even if for this kind of vessel a non-Newtonian model would be more appropriate. We used $\rho^{f}=1 \mathrm{~g} / \mathrm{cm}^{3}, \mu^{f}=0.03 \mathrm{cPa}$. Autoregulation is not included in this test. The reader interested in test cases including autoregulation is referred to [17]

Pressure conditions are applied at the inlet:

$$
P_{\text {in }}(t)[\mathrm{mmHg}]= \begin{cases}25.12 \sin (\pi t / 0.25)+45 & t \in[0,0.25] \\ 45 & t \in[0.25,0.8] .\end{cases}
$$

The mean value over time of the incoming pressure is $50 \mathrm{mmHg}$. At the outlets of the domain, the downstream circulation is connected to a venous pressure of $20 \mathrm{mmHg}$ via Windkessel compartments. More precisely, we use an RCR model where the parameters are $R_{\text {prox }}=6.10^{8} \mathrm{~Pa} \mathrm{~s} \mathrm{~cm}{ }^{-3}, R_{\text {dist }}=6.10^{9} \mathrm{~Pa} \mathrm{~s}$ $\mathrm{cm}^{-3}$ and the capacitance is $1.6710^{-8} \mathrm{~cm}^{3} \mathrm{~s}^{-1} \mathrm{~Pa}-1$. On the lateral surface an external pressure, modeling the intra-ocular pressure, of $15 \mathrm{mmHg}$ is also imposed.

The displacement of the arterial wall is shown in Fig.7 at the systolic peak and in Fig. 8 at the end of the diastolic phase. The fact that the displacement is smaller towards the end of the networkis mainly due to two reasons: the pressure at the end of the the network is significantly lower (the pressure drop is between 5 and $10 \mathrm{mmHg}$ ) and the diameter of the vessels is smaller. Indeed, the spring contribution to the structure is characterized by a coefficient that, at the first order approximation in $\eta$, depends on the 


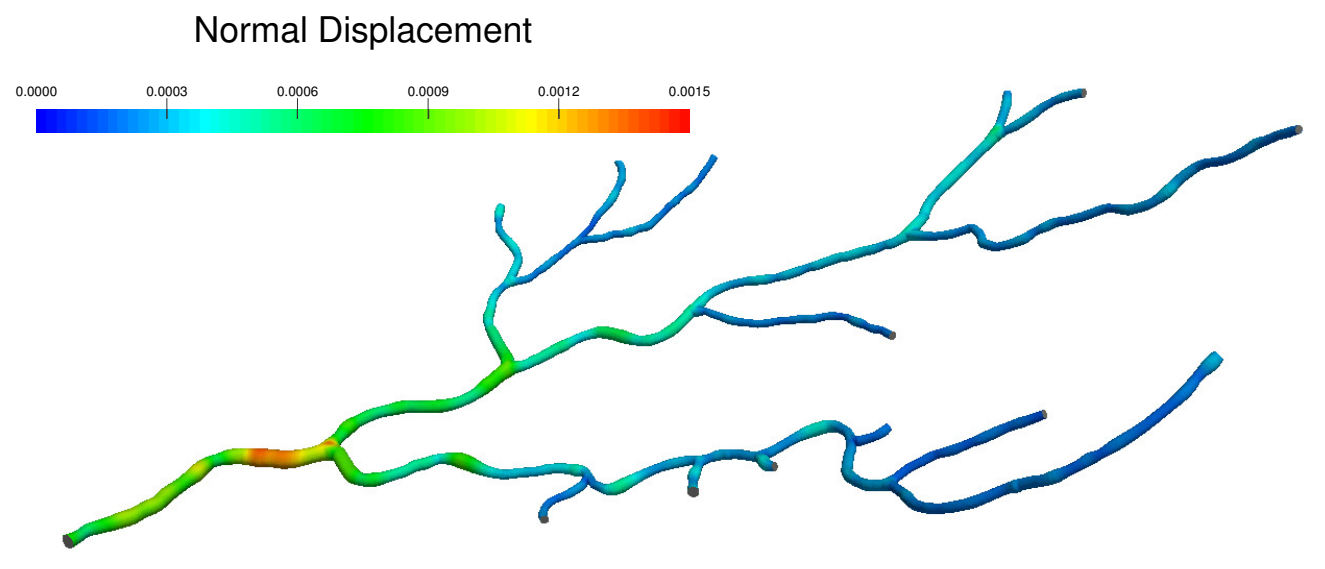

Figure 7: Displacement field on the retinal vasculature during the systolic peak. 


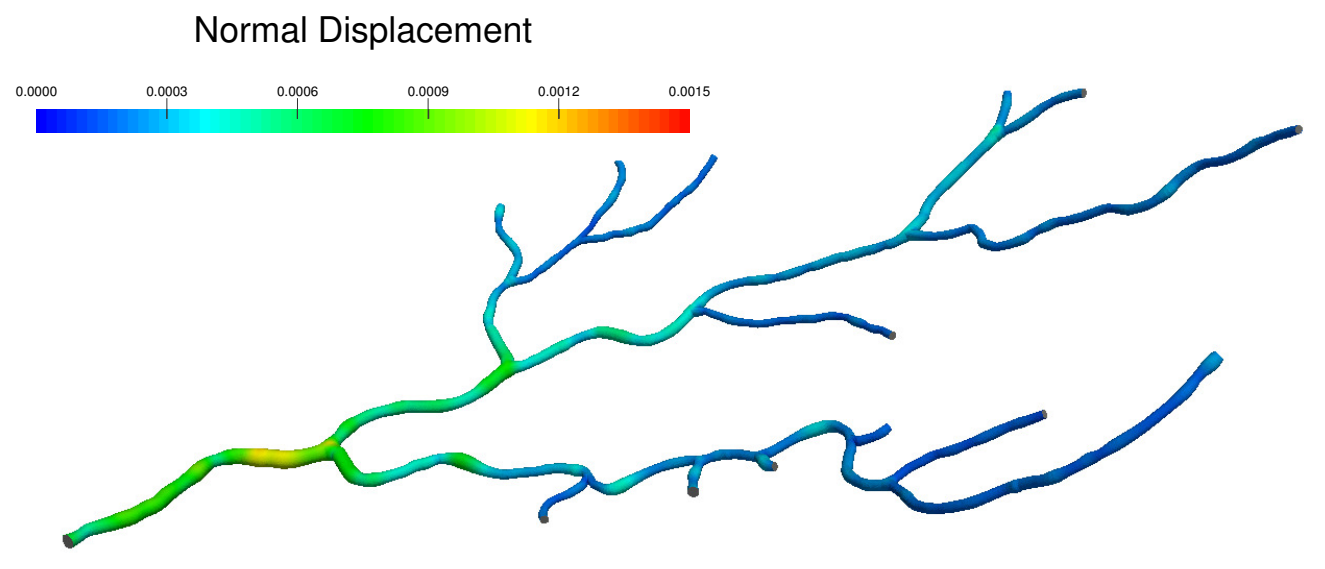

Figure 8: Displacement field on the retinal vasculature at the end of diastolic phase. 


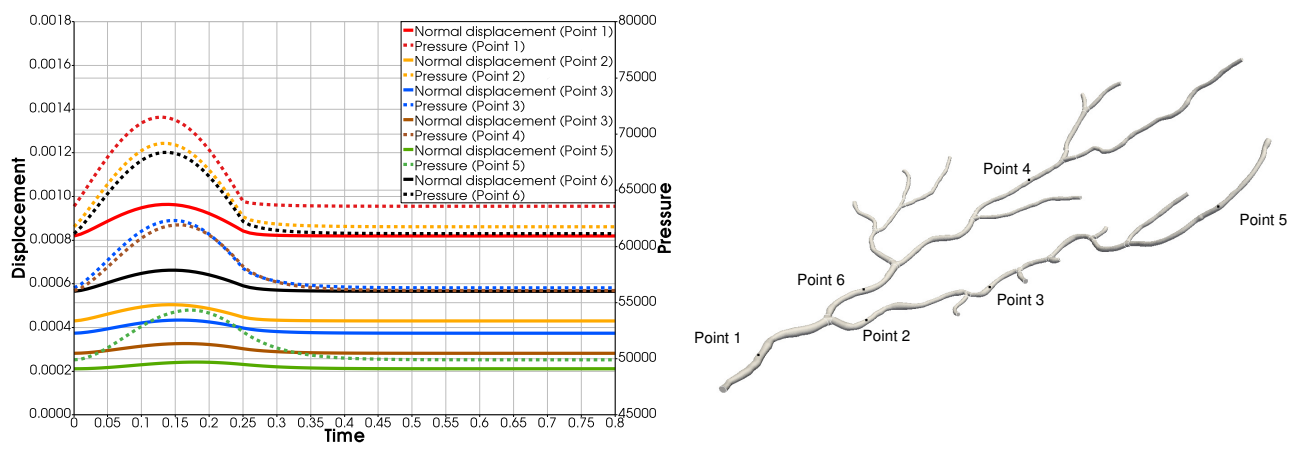

Figure 9: Left-hand side: the values of the displacement (solid lines, [cm], left axis) and pressure (dashed lines, $[\mathrm{Pa}]$, right axis) over time for six different points on the network. Right-hand side: the network of arterioles.

inverse of the vessel radius squared (as in the Laplace law). This can be quantitavely observed in Fig. 9 by comparing Point 2 and Point 6 (orange and black curves) or by comparing Point 3 and Point 4 . In these two cases, we observe similar pressure curves (dashed lines) and different displacement (solid lines).

In the same figure we also observe the propagation of the pressure wave through the vessel network: by comparing Point 1 with Point 5 (red and green curves) we see that the position of the pressure (and displacement) peak is delayed in Point 5 with respect to Point 1. On the other hand the amplitude of the wave is reduced, which is compatible with the fact that no pulsation is observed at the capillary level.

From a practical viewpoint, it is interesting to note that these fluidstructure results have been obtained at a computational cost similar to a fluid problem. It would be interesting to compare the results with those provided by more complex approaches, as was done in [13] for other simplified models. This will be the object of future work.

\section{Aknowledgements}

This research was made possible by a Marie Curie grant from the European Commission in the framework of the REVAMMAD ITN (Initial Training Research network), Project number 316990.

[1] T. B. Le, F. Sotiropoulos, Fluidstructure interaction of an aortic heart valve prosthesis driven by an animated anatomic left 
ventricle, Journal of Computational Physics 244 (0) (2013) 41 62, multi-scale Modeling and Simulation of Biological Systems. doi:http://dx.doi.org/10.1016/j.jcp.2012.08.036.

URL http://www. sciencedirect.com/science/article/pii/S0021999112004937

[2] R. van Loon, P. Anderson, J. de Hart, F. Baaijens, A combined fictitious domain/adaptative meshing method for fluid-structure interaction in heart valves, Int. J. Num. Meth. Fluids 46 (2004) 533-544.

[3] R. van Loon, P. Anderson, F. van de Vosse, A fluid-structure interaction method with solid-rigid contact for heart valve dynamics, J. Comp. Phys. 217 (2006) 806-823.

[4] M. Astorino, J.-F. Gerbeau, O. Pantz, K. Traoré, Fluid-structure interaction and multi-body contact. Application to aortic valves, Comp. Meth. Appl. Mech. Engng. 198 (2009) 3603-3612.

[5] Y. Bazilevs, V. Calo, Y. Zhang, T. J. Hughes, Isogeometric fluidstructure interaction analysis with applications to arterial blood flow, Computational Mechanics 38 (4-5) (2006) 310-322.

[6] P. Crosetto, P. Reymond, S. Deparis, D. Kontaxakis, N. Stergiopulos, A. Quarteroni, Fluid-structure interaction simulation of aortic blood flow, Computers \& Fluids 43 (1) (2011) 46-57.

[7] P. Moireau, N. Xiao, M. Astorino, C. A. Figueroa, D. Chapelle, C. A. Taylor, J.-F. Gerbeau, External tissue support and fluid-structure simulation in blood flows, Biomechanics and Modeling in Mechanobiology 11 (1) (2012) 1-18. doi:10.1007/s10237-011-0289-z.

[8] M. A. Fernández, M. Landajuela, M. Vidrascu, Fully decoupled timemarching schemes for incompressible fluid/thin-walled structure interaction, Journal of Computational Physics 297 (2015) 156-181.

[9] M. Bukač, S. Čanić, R. Glowinski, B. Muha, A. Quaini, A modular, operator-splitting scheme for fluid-structure interaction problems with thick structures, International journal for numerical methods in fluids 74 (8) (2014) 577-604.

[10] E. Burman, M. A. Fernández, Explicit strategies for incompressible fluid-structure interaction problems: Nitsche type mortaring versus 
robin-robin coupling, International Journal for Numerical Methods in Engineering 97 (10) (2014) 739-758.

[11] C. A. Figueroa, I. E. Vignon-Clementel, K. E. Jansen, T. J. Hughes, C. A. Taylor, A coupled momentum method for modeling blood flow in three-dimensional deformable arteries, Computer methods in applied mechanics and engineering 195 (41) (2006) 5685-5706.

[12] F. Nobile, C. Vergara, An effective fluid-structure interaction formulation for vascular dynamics by generalized robin conditions, SIAM Journal on Scientific Computing 30 (2) (2008) 731-763. arXiv:http://dx.doi.org/10.1137/060678439, doi:10.1137/060678439.

URL http://dx.doi.org/10.1137/060678439

[13] C. M. Colciago, S. Deparis, A. Quarteroni, Comparisons between reduced order models and full 3D models for fluid-structure interaction problems in haemodynamics, Journal of Computational and Applied Mathematics 265 (2014) 120-138.

[14] O. Pironneau, Simplified Fluid-Structure Interactions for Hemodynamics, in: S. R. Idelsohn (Ed.), Numerical Simulations of Coupled Problems in Engineering, Vol. 33 of Computational Methods in Applied Sciences, Springer International Publishing, 2014, pp. 57-70. doi:10.1007/978-3319-06136-8.

URL http://dx.doi.org/10.1007/978-3-319-06136-8

[15] T. Chacon-Rebollo, V. Girault, F. Murat, O. Pironneau, Analysis of a Simplified Coupled Fluid-Structure Model for Computational Hemodynamics, http://hal.upmc.fr/hal-01003208 (Apr. 2014).

[16] J. Arciero, A. Harris, B. Siesky, A. Amireskandari, V. Gershuny, A. Pickrell, G. Guidoboni, Theoretical analysis of vascular regulatory mechanisms contributing to retinal blood flow autoregulation, Investigative Ophthalmology \& Visual Science 54 (8) (2013) 55845593. arXiv:http://www.iovs.org/content/54/8/5584.full.pdf + html, doi:10.1167/iovs.12-11543.

URL http://www. iovs . org/content/54/8/5584 . abstract

[17] M. Aletti, J.-F. Gerbeau, D. Lombardi, Modeling autoregulation in three-dimensional simulations of retinal hemodynamics, Journal for Modeling in Ophthalmology 1 (1) (2016) 88-115. 
[18] P. G. Ciarlet, Mathematical elasticity. Vol. III, Vol. 29 of Studies in Mathematics and its Applications, North-Holland Publishing Co., Amsterdam, 2000, theory of shells.

[19] K. Gostaf, O. Pironneau, Pressure Boundary Conditions for Blood Flows, http://hal.upmc.fr/hal-00865671 (Sep. 2013).

[20] P. Causin, J.-F. Gerbeau, F. Nobile, Added-mass effect in the design of partitioned algorithms for fluid-structure problems, Comput. Methods Appl. Mech. Engrg. 194 (42-44) (2005) 4506-4527.

[21] J. Staal, M. Abramoff, M. Niemeijer, M. Viergever, B. van Ginneken, Ridge based vessel segmentation in color images of the retina, IEEE Transactions on Medical Imaging 23 (4) (2004) 501-509.

[22] B. Al-Diri, A. Hunter, D. Steel, An active contour model for segmenting and measuring retinal vessels, Medical Imaging, IEEE Transactions on 28 (9) (2009) 1488-1497.

[23] F. Caliva, M. Aletti, B. Al-Diri, A. Hunter, A new tool to connect blood vessels in fundus retinal images, in: Engineering in Medicine and Biology Society (EMBC), 2015 37th Annual International Conference of the IEEE, IEEE, 2015, pp. 4343-4346.

[24] G. Guidoboni, A. Harris, S. Cassani, J. Arciero, B. Siesky, A. Amireskandari, L. Tobe, P. Egan, I. Januleviciene, J. Park, Intraocular pressure, blood pressure, and retinal blood flow autoregulation: A mathematical model to clarify their relationship and clinical relevance, Investigative ophthalmology \& visual science 55 (7) (2014) 4105.

[25] C. J. Pournaras, E. Rungger-Brändle, C. E. Riva, S. H. Hardarson, E. Stefansson, Regulation of retinal blood flow in health and disease, Progress in retinal and eye research 27 (3) (2008) 284-330. 


\section{Appendix A. Derivation of the nonlinear elastic energy}

In this Appendix, the expression of the elastic energy for the non-linear Koiter model is derived, when the simplifying hypotheses for the problem (see Section 2.2) are taken into account.

We refer to Section 2.1 for the differential geometry notation.

The expression of the energy functional, when $\boldsymbol{f}=\mathbf{0}$,

$$
\psi^{\kappa}(\boldsymbol{\eta})=\frac{1}{2} \int_{\omega} \mathcal{E}^{\alpha \beta \sigma \tau} g_{\sigma \tau}(\boldsymbol{\eta}) g_{\alpha \beta}(\boldsymbol{\eta}) h_{\kappa} \sqrt{a} d \boldsymbol{\xi}
$$

is written in terms of the elasticity tensor defined on the surface and of the change of metric tensor. The elasticity tensor, $\mathcal{E}^{\alpha \beta \sigma \tau}$, depends on the Lamé coefficients $\lambda^{s}$ and $\mu^{s}$ and on inverse of the first fundamental form, $\boldsymbol{A}^{-1}$, of the surface. In order to simplify the calculations the term $\mathcal{E}^{\alpha \beta \sigma \tau} g_{\sigma \tau}(\boldsymbol{\eta}) g_{\alpha \beta}(\boldsymbol{\eta}) h_{\kappa}$ is split into two different contributions:

$$
\begin{aligned}
\mathcal{E}^{\alpha \beta \sigma \tau} g_{\sigma \tau}(\boldsymbol{\eta}) g_{\alpha \beta}(\boldsymbol{\eta}) & =\underbrace{\frac{4 \lambda^{s} \mu^{s}}{\lambda^{s}+2 \mu^{s}} a^{\alpha \beta} a^{\sigma \tau} g_{\sigma \tau}(\boldsymbol{\eta}) g_{\alpha \beta}(\boldsymbol{\eta})}_{I} \\
& +\underbrace{2 \mu^{s} a^{\alpha \sigma} a^{\beta \tau} g_{\sigma \tau}(\boldsymbol{\eta}) g_{\alpha \beta}(\boldsymbol{\eta})+2 \mu^{s} a^{\alpha \tau} a^{\beta \sigma} g_{\sigma \tau}(\boldsymbol{\eta}) g_{\alpha \beta}(\boldsymbol{\eta})}_{I I} .
\end{aligned}
$$

In the following the dependence of $\boldsymbol{G}$ on $\boldsymbol{\eta}$ is dropped for sake of compactness in the notation. Since $\boldsymbol{A}, \boldsymbol{B}, \boldsymbol{G}$ are symmetric, the two contributions can be further simplified and written in a more compact form as functions of the tensor $\boldsymbol{A}^{-1} \boldsymbol{G}$ :

$$
\begin{gathered}
I=\frac{4 \lambda^{s} \mu^{s}}{\lambda^{s}+2 \mu^{s}}\left(a^{\alpha \beta} g_{\alpha \beta}\right)^{2}=\frac{4 \lambda^{s} \mu^{s}}{\lambda^{s}+2 \mu^{s}}\left(\operatorname{tr}\left(\boldsymbol{A}^{-1} \boldsymbol{G}\right)\right)^{2} \\
I I=4 \mu^{s} a^{\alpha \sigma} g_{\sigma \tau} a^{\tau \beta} g_{\beta \alpha}=4 \mu^{s} \operatorname{tr}\left(\left(\boldsymbol{A}^{-1} \boldsymbol{G}\right)^{2}\right) .
\end{gathered}
$$

By injecting Eq.(A.1) and (A.2) into the expression of the energy functional, 
the following is obtained:

$$
\begin{aligned}
\psi^{\kappa}(\eta) & =\frac{1}{4} \int_{\Gamma} h^{\kappa} \mathcal{E}^{\alpha \beta \sigma \tau} g_{\sigma \tau} g_{\alpha \beta} \sqrt{a} d \boldsymbol{\xi} \\
& =\frac{1}{4} \int_{\Gamma} h^{\kappa}\left[\frac{4 \lambda^{s} \mu^{s}}{\lambda^{s}+2 \mu^{s}} \operatorname{tr}\left(\boldsymbol{A}^{-1} \boldsymbol{G}\right)^{2}+4 \mu^{s} \operatorname{tr}\left(\left(\boldsymbol{A}^{-1} \boldsymbol{G}\right)^{2}\right)\right] \sqrt{a} d \boldsymbol{\xi} \\
& =\frac{1}{4} \int_{\Gamma} h^{\kappa}\left[\frac{2 E \nu}{1-\nu^{2}} \operatorname{tr}\left(\boldsymbol{A}^{-1} \boldsymbol{G}\right)^{2}+\frac{2 E}{1+\nu} \operatorname{tr}\left(\left(\boldsymbol{A}^{-1} \boldsymbol{G}\right)^{2}\right)\right] \sqrt{a} d \boldsymbol{\xi} \\
& =\frac{1}{2} \int_{\Gamma} \frac{h^{\kappa} E}{1-\nu^{2}}\left[\nu \operatorname{tr}\left(\boldsymbol{A}^{-1} \boldsymbol{G}\right)^{2}+(1-\nu) \operatorname{tr}\left(\left(\boldsymbol{A}^{-1} \boldsymbol{G}\right)^{2}\right)\right] \sqrt{a} d \boldsymbol{\xi},
\end{aligned}
$$

where the relationships between the Lamè coefficients $\mu^{s}, \lambda^{s}$ and the Young modulus and the Poisson ratio are used, namely:

$$
\lambda^{s}=\frac{E \nu}{(1+\nu)(1-2 \nu)}, \mu^{s}=\frac{E}{2(1+\nu)} .
$$

The expression of the change of metric tensor is rewritten, by considering that the displacement field is, by hypothesis, aligned with the outward normal:

$$
g_{\alpha \beta}=-b_{\alpha \beta} \eta+\frac{1}{2} s_{\tau \alpha} b_{\tau \beta} \eta^{2}+\frac{1}{2} \partial_{\alpha} \eta \partial_{\beta} \eta
$$

where $s_{\tau \alpha}$ denotes the components of the matrix representation of $\boldsymbol{S}$.

In order to expand the terms in Eq.(A.3) in terms of $\eta$, we write the components of the tensor $\boldsymbol{A}^{-1} \boldsymbol{G}$

$$
g_{\beta}^{\delta}=\left(\boldsymbol{A}^{-1} \boldsymbol{G}\right)_{\delta \beta}=a^{\delta \alpha} g_{\alpha \beta}=-a^{\delta \alpha} b_{\alpha \beta} \eta+\frac{1}{2} a^{\delta \alpha} a^{\sigma \tau} b_{\sigma \alpha} b_{\tau \beta} \eta^{2}+\frac{1}{2} a^{\delta \alpha} \partial_{\alpha} \eta \partial_{\beta} \eta .
$$

By introducing the notation $\partial^{\alpha}=a^{\alpha \sigma} \partial_{\sigma}, g_{\beta}^{\delta}$ it simplifies to

$$
g_{\beta}^{\delta}=-s_{\delta \beta} \eta+\frac{1}{2} s_{\delta \sigma} s_{\sigma \beta} \eta^{2}+\frac{1}{2} \partial^{\delta} \eta \partial_{\beta} \eta .
$$

After some algebraic calculations the quantities $\operatorname{tr}\left(\boldsymbol{A}^{-1} \boldsymbol{G}\right)$ and $\operatorname{tr}\left(\left(\boldsymbol{A}^{-1} \boldsymbol{G}\right)^{2}\right)$ can be written in terms of traces of powers of $\boldsymbol{S}$, which are, in turn, directly related to the curvatures of the surfaces.

It holds:

$$
\begin{aligned}
\operatorname{tr}\left(\boldsymbol{A}^{-1} \boldsymbol{G}\right)= & -\operatorname{tr}(\boldsymbol{S}) \eta+\frac{1}{2} \operatorname{tr}\left(\boldsymbol{S}^{2}\right) \eta^{2}+\frac{1}{2} \nabla \eta^{T} \boldsymbol{A}^{-1} \nabla \eta \\
\operatorname{tr}\left(\left(\boldsymbol{A}^{-1} \boldsymbol{G}\right)^{2}\right)= & \operatorname{tr}\left(\boldsymbol{S}^{2}\right) \eta^{2}-\operatorname{tr}\left(\boldsymbol{S}^{3}\right) \eta^{3}-\eta \nabla \eta^{T} \boldsymbol{S} \boldsymbol{A}^{-1} \nabla \eta \\
& +\frac{1}{4} \eta^{4} \operatorname{tr}\left(\boldsymbol{S}^{4}\right)+\frac{1}{2} \eta^{2} \nabla \eta \boldsymbol{S}^{2} \boldsymbol{A}^{-1} \nabla \eta+\frac{1}{4}\left(\nabla \eta^{T} \boldsymbol{A}^{-1} \nabla \eta\right)^{2}
\end{aligned}
$$


The traces of powers of $\boldsymbol{S}$ can be written in terms of the curvatures as:

$$
\begin{aligned}
& \operatorname{tr}(\boldsymbol{S})=\lambda_{1}+\lambda_{2}=2 \rho_{1} \\
& \operatorname{tr}\left(\boldsymbol{S}^{2}\right)=\lambda_{1}^{2}+\lambda_{2}^{2}=4 \rho_{1}^{2}-2 \rho_{2} \\
& \operatorname{tr}\left(\boldsymbol{S}^{3}\right)=\lambda_{1}^{3}+\lambda_{2}^{3}=8 \rho_{1}^{3}-6 \rho_{1} \rho_{2} \\
& \operatorname{tr}\left(\boldsymbol{S}^{4}\right)=\lambda_{1}^{4}+\lambda_{2}^{4}=16 \rho_{1}^{4}+2 \rho_{2}^{2}-16 \rho_{1}^{2} \rho_{2},
\end{aligned}
$$

where $\rho_{1}$ is the mean curvature $1 / 2\left(\lambda_{1}+\lambda_{2}\right)$ and $\rho_{2}$ is the Gaussian curvature $\lambda_{1} \lambda_{2}$.

By using Eq.(A.4) we derive the final expression for the elastic energy functional

$$
\begin{aligned}
\psi^{\kappa}(\eta)= & \frac{1}{4} \int_{\omega} h^{\kappa} \mathcal{E}^{\alpha \beta \sigma \tau} g_{\sigma \tau} g_{\alpha \beta} \sqrt{a} d \boldsymbol{\xi} \\
= & \frac{1}{2} \int_{\omega} \frac{h^{\kappa} E}{\left(1-\nu^{2}\right)}\left[\nu \operatorname{tr}\left(\boldsymbol{A}^{-1} \boldsymbol{G}\right)^{2}+(1-\nu) \operatorname{tr}\left(\left(\boldsymbol{A}^{-1} \boldsymbol{G}\right)^{2}\right)\right] \sqrt{a} d \boldsymbol{\xi} \\
= & \frac{1}{2} \int_{\omega} \frac{h^{\kappa} E}{\left(1-\nu^{2}\right)}\left[c_{1} \eta^{2}-2 c_{2} \eta^{3}+c_{3} \eta^{4}+\frac{1}{4}\left((\nabla \eta)^{T} \boldsymbol{A}^{-1} \nabla \eta\right)^{2}+\right. \\
& -2 \nu \rho_{1} \eta(\nabla \eta)^{T} \boldsymbol{A}^{-1} \nabla \eta+\nu\left(2 \rho_{1}^{2}-\rho_{2}\right) \eta^{2}(\nabla \eta)^{T} \boldsymbol{A}^{-1} \nabla \eta+ \\
& \left.-(1-\nu) \eta(\nabla \eta)^{T} \boldsymbol{S} \boldsymbol{A}^{-1} \nabla \eta+\frac{1}{2}(1-\nu) \eta^{2}(\nabla \eta)^{T} \boldsymbol{S}^{2} \boldsymbol{A}^{-1} \nabla \eta\right] \sqrt{a} d \boldsymbol{\xi},
\end{aligned}
$$

where the coefficients $c_{j}$ depend on the Poisson ratio and on the curvatures (they are reported in Eq.(4)).

In order to compute the form $\Psi^{\kappa}$ in Eq.(2), the first variation with respect to $\eta$ of the energy functional has to be computed and tested, to get the weak form, against a function $\chi$ belonging to a suitable functional space. The procedure to derive the first variation (Fréchet derivative) involves several integrations by parts on the surface that produce extra terms involving the curvatures. These terms, however, disappear when the first variation is tested against the function $\chi$. The final result is reported in Eq.(3).

\section{Appendix B. Details on the time discretization of the boundary condition}

The nonlinear form $\Psi^{s}(\eta, \chi)$ depends on the forms associated to the nonlinear Koiter shell and the fibers (see Eq.(7)). For the sake of simplicity, we 
consider only fibers in the $\boldsymbol{w}$ direction.

$$
\begin{aligned}
& \Psi^{s}(\eta, \chi)=\frac{2 E}{1-\nu^{2}} \int_{\omega}\left(c_{1} \eta-3 c_{2} \eta^{2}+2 c_{3} \eta^{3}\right) \chi-2 \nabla \chi^{T}\left(C_{1} \eta+C_{2} \eta^{2}\right) \nabla \eta+(\mathrm{B} .1) \\
& -\nabla^{T} \eta\left[\left(C_{1}+2 C_{2} \eta\right) \chi\right] \nabla \eta+\frac{1}{2}\left(\nabla \eta^{T} A^{-1} \nabla \eta\right) \nabla^{T} \chi A^{-1} \nabla \eta h_{\kappa} \sqrt{a} d \boldsymbol{\xi}- \\
& \int_{\omega} \boldsymbol{f}_{n} \cdot \chi h_{\kappa} \sqrt{a} d \boldsymbol{\xi}+\int_{\omega} \varrho_{\mathbf{w}} \nabla \chi^{T}\left[k_{0}+k_{1}\left(-d_{1}+\frac{d_{2}}{2} \eta^{2}\right)+\frac{k_{1}}{4} W\right] P_{\mathbf{w}} \nabla \eta+ \\
& \varrho_{\mathbf{w}}\left[k_{0} d_{2} \eta+k_{1}\left(-d_{1}^{2} \eta-\frac{3 d_{1} d_{2}}{2} \eta^{2}+\frac{d_{2}^{2}}{2} \eta^{3}\right)+\frac{k_{1}}{2}\left(-d_{1}+d_{2} \eta\right) W\right] \chi h_{f} \sqrt{a} d \boldsymbol{\xi} .
\end{aligned}
$$

We use a semi-implicit approach to discretize this form in time. The scheme is the following

$$
\begin{aligned}
& \Psi^{s}\left(\eta^{k+1}, \chi ; \eta^{k}\right)=\frac{2 E}{1-\nu^{2}} \int_{\omega}\left(c_{1}-3 c_{2} \eta^{k}+2 c_{3} \eta^{k^{2}}\right) \eta^{k+1} \chi-2 \nabla \chi^{T}\left(C_{1} \eta^{k}+C_{2} \eta^{k^{2}}\right) \nabla \eta^{k+1}+ \\
& -\nabla^{T} \eta^{k}\left[\left(C_{1}+2 C_{2} \eta^{k+1}\right) \chi\right] \nabla \eta^{k}+\frac{1}{2}\left(\nabla \eta^{k^{T}} A^{-1} \nabla \eta^{k}\right) \nabla^{T} \chi A^{-1} \nabla \eta^{k+1} h_{\kappa} \sqrt{a} d \boldsymbol{\xi}- \\
& \int_{\omega} \boldsymbol{f}_{n}^{k+1} \cdot \chi h_{\kappa} \sqrt{a} d \boldsymbol{\xi}+\int_{\omega} \varrho_{\mathbf{w}} \nabla \chi^{T}\left[k_{0}+k_{1}\left(-d_{1}+\frac{d_{2}}{2} \eta^{k^{2}}\right)+\frac{k_{1}}{4} W^{k}\right] P_{\mathbf{w}} \nabla \eta^{k+1}+ \\
& \varrho_{\mathbf{w}}\left[k_{0} d_{2} \eta^{k+1}+k_{1}\left(-d_{1}^{2}-\frac{3 d_{1} d_{2}}{2} \eta^{k}+\frac{d_{2}^{2}}{2} \eta^{k^{2}}\right) \eta^{k+1}+\frac{k_{1}}{2}\left(-d_{1}+d_{2} \eta^{k+1}\right) W^{k}\right] \chi h_{f} \sqrt{a} d \boldsymbol{\xi} .
\end{aligned}
$$

This form is linear with respect to $\chi$ and affine with respect to $\eta^{k+1}$. The coefficients of the form depend on the current displacement $\eta^{k}$. It is useful to split the form into two sub-contributions in order to highlight the bilinear part

$$
\Psi^{s}\left(\eta^{k+1}, \chi ; \eta^{k}\right)=\mathcal{B}\left(\eta^{k+1}, \chi ; \eta^{k}\right)+\mathcal{G}\left(\chi ; \eta^{k}\right)
$$


The bilinear form $\mathcal{B}\left(\eta^{k+1}, \chi ; \eta^{k}\right)$ has the following expression:

$$
\begin{aligned}
& \mathcal{B}\left(\eta^{k+1}, \chi ; \eta^{k}\right)=\frac{2 E}{1-\nu^{2}} \int_{\omega}\left(c_{1}-3 c_{2} \eta^{k}+2 c_{3} \eta^{k^{2}}-2\left(\nabla^{T} \eta^{k} C_{2} \nabla \eta^{k}\right)\right) \eta^{k+1} \chi \\
& -2 \nabla \chi^{T}\left(C_{1} \eta^{k}+C_{2} \eta^{k^{2}}\right) \nabla \eta^{k+1}+\frac{1}{2}\left(\nabla \eta^{k^{T}} A^{-1} \nabla \eta^{k}\right) \nabla^{T} \chi A^{-1} \nabla \eta^{k+1} h_{\kappa} \sqrt{a} d \boldsymbol{\xi}+ \\
& +\int_{\omega} \varrho_{\mathbf{w}} \nabla \chi^{T}\left[k_{0}+k_{1}\left(-d_{1}+\frac{d_{2}}{2} \eta^{k^{2}}\right)+\frac{k_{1}}{4} W^{k}\right] P_{\mathbf{w}} \nabla \eta^{k+1}+ \\
& \varrho_{\mathbf{w}}\left[k_{0} d_{2}+k_{1}\left(-d_{1}^{2}-\frac{3 d_{1} d_{2}}{2} \eta^{k}+\frac{d_{2}^{2}}{2} \eta^{k^{2}}\right)+\frac{k_{1}}{2} d_{2} W^{k}\right] \eta^{k+1} \chi h_{f} \sqrt{a} d \boldsymbol{\xi} .
\end{aligned}
$$

On the other hand, the functional $\mathcal{G}\left(\chi ; \eta^{k}\right)$ has the form

$$
\begin{gathered}
\mathcal{G}\left(\chi ; \eta^{k}\right)=-\frac{2 E}{1-\nu^{2}} \int_{\omega}\left(\nabla^{T} \eta^{k} C_{1} \nabla \eta^{k}\right) \chi h_{\kappa} \sqrt{a} d \boldsymbol{\xi}- \\
\int_{\omega} \boldsymbol{f}_{n}^{k+1} \cdot \chi h_{\kappa} \sqrt{a} d \boldsymbol{\xi}-\int_{\omega} \varrho_{\mathbf{w}} \frac{k_{1}}{2} d_{1} W^{k} \chi h_{f} \sqrt{a} d \boldsymbol{\xi} .
\end{gathered}
$$

We use the strong formulation of Eq.(11),

$$
\eta^{k+1}=\left(1+\Delta t \nabla_{\mathbf{n n}} \boldsymbol{u}^{k}\right) \eta^{k}+\Delta t u_{\mathbf{n}}^{k+1}
$$

to replace $\eta^{k+1}$ in Eq.(B.2) obtaining:

$$
\Psi^{s}\left(\eta^{k+1}, \chi ; \eta^{k}\right)=\Delta t \mathcal{B}\left(u_{\mathbf{n}}^{k+1}, \chi ; \eta^{k}\right)+\mathcal{B}\left(\left(1+\Delta t \nabla_{\mathbf{n n}} \boldsymbol{u}^{k}\right) \eta^{k}, \chi ; \eta^{k}\right)+\mathcal{G}\left(\chi ; \eta^{k}\right)
$$

which is in the same form as Eq.(13). We, finally, define:

$$
\begin{aligned}
& \Phi^{k+1}\left(u_{\mathbf{n}}^{k+1}, \chi\right)=\Delta t \mathcal{B}\left(u_{\mathbf{n}}^{k+1}, \chi ; \eta^{k}\right) \\
& \phi^{k}(\chi)=\mathcal{B}\left(\left(1+\Delta t \nabla_{\mathbf{n n}} \boldsymbol{u}^{k}\right) \eta^{k}, \chi ; \eta^{k}\right)+\mathcal{G}\left(\chi ; \eta^{k}\right)
\end{aligned}
$$

The expression for $\mathcal{F}$ in equation (14) is obtained by combining Eq.(B.4) and Eq.(12).

$$
\mathcal{F}=\left\langle\eta^{k} \nabla_{\mathbf{n}} p^{k}-p^{r e f}-\frac{\rho_{s} h_{s}}{\Delta t^{2}}\left(\left(\Delta t \nabla_{\mathbf{n n}} \boldsymbol{u}^{k}-1\right) \eta^{k}+\eta^{k-1}\right), \chi\right\rangle_{\omega}-\phi^{k}(\chi)
$$


On the interface surface $\Gamma$ those two boundary conditions (Eq.(10), Eq.(14)) hold:

$$
\left\{\begin{array}{l}
\left\langle(\boldsymbol{I}-\boldsymbol{n} \otimes \boldsymbol{n}) \boldsymbol{u}^{k+1}, \boldsymbol{w}\right\rangle_{\omega}=-\left\langle\eta^{k}(\boldsymbol{I}-\boldsymbol{n} \otimes \boldsymbol{n}) \nabla \boldsymbol{u}^{k} \boldsymbol{n}, \boldsymbol{w}\right\rangle_{\omega} \\
\frac{\rho^{s} h^{s}}{\Delta t}\left\langle u_{\boldsymbol{n}}^{k+1}, \chi\right\rangle_{\omega}+\Phi^{k+1}\left(u_{\boldsymbol{n}}^{k+1}, \chi\right)-\left\langle p^{k+1}, \chi\right\rangle_{\omega}=\mathcal{F}\left(\chi ; \eta^{k}, p^{k}, u_{\boldsymbol{n}}^{k}\right) .
\end{array}\right.
$$

Let $\mathbf{z}: \Gamma \mapsto \mathbb{R}^{3}$ be a vector-valued function of $\mathbf{H}^{1}(\Gamma)$, such that $\boldsymbol{z} \cdot \boldsymbol{n}=\chi$ and $(\boldsymbol{I}-\boldsymbol{n} \otimes \boldsymbol{n}) \boldsymbol{z}=\boldsymbol{w}$. We also recall that $\boldsymbol{w} \cdot \boldsymbol{n}=0$. By introducing the notation $\boldsymbol{T}=(\boldsymbol{I}-\boldsymbol{n} \otimes \boldsymbol{n})$ and by reorganizing the terms $\frac{\rho^{s} h^{s}}{\Delta t}\left\langle u_{\boldsymbol{n}}^{k+1}, \chi\right\rangle_{\omega}+\Phi^{k+1}\left(u_{\boldsymbol{n}}^{k+1}, \chi\right)$ we get

$$
\left\{\begin{array}{l}
\left\langle\boldsymbol{T} \boldsymbol{u}^{k+1}, \boldsymbol{w}\right\rangle_{\omega}=-\left\langle\eta^{k} \boldsymbol{T} \nabla \boldsymbol{u}^{k} \boldsymbol{n}, \boldsymbol{w}\right\rangle_{\omega} \\
\left\langle\alpha^{k} u_{\boldsymbol{n}}^{k+1}, \chi\right\rangle_{\omega}+\widetilde{\Phi}^{k+1}\left(u_{\boldsymbol{n}}^{k+1}, \chi\right)-\left\langle p^{k+1}, \chi\right\rangle_{\omega}=\mathcal{F}\left(\chi ; \eta^{k}, p^{k}, u_{\boldsymbol{n}}^{k}\right)
\end{array}\right.
$$

where

$$
\begin{array}{r}
\alpha^{k}=\frac{\rho^{s} h^{s}}{\Delta t}+\frac{2 E \Delta t h_{\kappa}}{1-\nu^{2}}\left(c_{1}-3 c_{2} \eta^{k}+2 c_{3} \eta^{k^{2}}-2\left(\nabla^{T} \eta^{k} C_{2} \nabla \eta^{k}\right)\right. \\
+\varrho_{\mathbf{w}} h_{f}\left[k_{0} d_{2}+k_{1}\left(-d_{1}^{2}-\frac{3 d_{1} d_{2}}{2} \eta^{k}+\frac{d_{2}^{2}}{2} \eta^{k^{2}}\right)+\frac{k_{1}}{2} d_{2} W^{k}\right]
\end{array}
$$

and

$$
\begin{aligned}
& \widetilde{\Phi}^{k+1}=\frac{2 E \Delta t}{1-\nu^{2}} \int_{\omega}-2 \nabla \chi^{T}\left(C_{1} \eta^{k}+C_{2} \eta^{k^{2}}\right) \nabla u_{\boldsymbol{n}}^{k+1}+\frac{1}{2}\left(\nabla \eta^{k^{T}} A^{-1} \nabla \eta^{k}\right) \nabla^{T} \chi A^{-1} \nabla u_{\boldsymbol{n}}^{k+1} h_{\kappa} \sqrt{a} d \boldsymbol{\xi}+ \\
& +\int_{\omega} \varrho_{\mathbf{w}} \Delta t \nabla \chi^{T}\left[k_{0}+k_{1}\left(-d_{1}+\frac{d_{2}}{2} \eta^{k^{2}}\right)+\frac{k_{1}}{4} W^{k}\right] P_{\mathbf{w}} \nabla u_{\boldsymbol{n}}^{k+1} h_{f} \sqrt{a} d \boldsymbol{\xi}
\end{aligned}
$$

Finally, we replace $\boldsymbol{w}$ by $\boldsymbol{T} \boldsymbol{z}$ and $\chi$ by $\boldsymbol{z} \cdot \boldsymbol{n}$, we multiply the equation for the tangential component by $\alpha^{k}$ and we sum the result

$$
\underbrace{\left\langle\alpha^{k} \boldsymbol{u}^{k+1}, \boldsymbol{z}\right\rangle_{\omega}+\widetilde{\Phi}^{k+1}\left(u_{\boldsymbol{n}}^{k+1}, \boldsymbol{z} \cdot \boldsymbol{n}\right)}_{\ell\left(\boldsymbol{u}^{k+1}, \boldsymbol{z}\right)}-\left\langle p^{k+1}, \boldsymbol{z} \cdot \boldsymbol{n}\right\rangle_{\omega}=\underbrace{\mathcal{F}\left(\boldsymbol{z} \cdot \boldsymbol{n} ; \eta^{k}, p^{k}, u_{\boldsymbol{n}}^{k}\right)-\left\langle\eta^{k} \boldsymbol{T} \nabla \boldsymbol{u}^{k} \boldsymbol{n}, \boldsymbol{T} \boldsymbol{z}\right\rangle_{\omega}}_{\mathcal{R}(\boldsymbol{z})} .
$$

